\title{
On magnetostrophic mean-field solutions of the geodynamo equations. Part 2
}

\author{
Paul H. Roberts ${ }^{1}$ and Cheng-Chin $\mathrm{Wu}^{1, \dagger}$ \\ ${ }^{1}$ Department of Mathematics, University of California, Los Angeles, CA 90095, USA
}

(Received 2 March 2018; revised 10 May 2018; accepted 21 May 2018)

\begin{abstract}
A dynamo driven by motions unaffected by viscous forces is termed 'magnetostrophic', but cannot be found through today's numerical simulations, which require substantial viscosity to stabilize solutions of the full magnetohydrodynamic (MHD) dynamo equations. By using an alternative numerical technique, proposed by Taylor (Proc. R. Soc. Lond. A, vol. 274, 1963, pp. 274-283), we recently obtained the first magnetostrophic dynamo solutions ever derived (Wu \& Roberts, Geophys. Astrophys. Fluid Dyn., vol. 109, 2014, pp. 84-110). These were axisymmetric and of mean-field type. In an earlier paper (Roberts \& Wu, Geophys. Astrophys. Fluid Dyn., vol. 108, 2014, pp. 696-715), we proposed an extension of Taylor's method. Here we explore its numerical implications, comparing them to the consequences of Taylor's original proposal. One of the differences between the two approaches is that our modification retains torsional waves but Taylor's theory does not. A more important difference is that our extension of Taylor's method is, for reasons presented here, the most general possible that does not suffer from the limitations imposed by viscosity on today's numerical simulations.
\end{abstract}

Key words: astrophysical plasmas, plasma flows, plasma nonlinear phenomena

\section{Introduction}

\subsection{Brief history of mean-field models}

This section gives our view of the history and development of mean-field dynamos, and prepares the ground for new material presented later.

The mean-field dynamo was the invention of Parker (1955). By the late 70s, his idea had blossomed into an entire subject, reviewed by Parker (1979) and by this special issue of Journal of Plasma Physics. The mean-field dynamo depends on a modification of Ohm's law for the electric current density, which in pre-Maxwell theory is $\boldsymbol{J}=$ $\sigma(\boldsymbol{E}+\boldsymbol{U} \times \boldsymbol{B})$, where $\boldsymbol{B}$ is the magnetic field, $\sigma$ is the electrical conductivity of the fluid and $\boldsymbol{U}$ is its velocity. Ampère's law relates $\boldsymbol{J}$ and $\boldsymbol{B}$ by $\boldsymbol{J}=\boldsymbol{\nabla} \times(\boldsymbol{B} / \mu)$, where $\mu$ is the magnetic permeability of the fluid, which is here taken to be that of free space: $\mu=4 \pi \times 10^{-7} \mathrm{H} \mathrm{m}^{-1}$. It follows that $\eta \nabla \times \boldsymbol{B}=\boldsymbol{E}+\boldsymbol{U} \times \boldsymbol{B}$, where $\eta=1 / \mu \sigma$ is the magnetic diffusivity.

Parker argued that, if the conductor is in turbulent motion, Ohm's law changes to $\overline{\boldsymbol{J}}=\sigma^{\mathrm{T}}(\overline{\boldsymbol{E}}+\overline{\boldsymbol{U}} \times \overline{\boldsymbol{B}}+\mathcal{E})$, where $\sigma^{\mathrm{T}}$ is the turbulent conductivity and $\mathcal{E}=\overline{\boldsymbol{u} \times \boldsymbol{b}}$, the overbar signifying the ensemble average of what is under it. Here $\boldsymbol{j}=\boldsymbol{J}-\overline{\boldsymbol{J}}, \boldsymbol{e}=\boldsymbol{E}-\overline{\boldsymbol{E}}$,

$\dagger$ Email address for correspondence: ccwu@ucla.edu 
$\boldsymbol{u}=\boldsymbol{U}-\overline{\boldsymbol{U}}, \boldsymbol{b}=\boldsymbol{B}-\overline{\boldsymbol{B}}$, etc. are the 'fluctuating parts' of $\boldsymbol{J}, \boldsymbol{E}, \boldsymbol{U}$ and $\boldsymbol{B}$; see Moffatt (1978, p. 148). In short, Parker (1957) posited that $\eta^{\mathrm{T}} \nabla \times \overline{\boldsymbol{B}}=\overline{\boldsymbol{E}}+\overline{\boldsymbol{U}} \times \overline{\boldsymbol{B}}+\mathcal{E}$, where $\eta^{\mathrm{T}}=1 / \mu \sigma^{\mathrm{T}}$ is the turbulent magnetic diffusivity, and $\eta^{\mathrm{T}} \gg \eta$. That $\sigma^{\mathrm{T}}$ might be much less than $\sigma$ had already been anticipated by Sweet (1950).

In principle, $\mathcal{E}$ can be found by integrating the full MHD equations, and taking the statistical average. This task is as difficult as any in theoretical physics. Therefore approximations to $\mathcal{E}$ are sought, motivated by a qualitative understanding of the fluid dynamics controlling $\boldsymbol{U}$. The simplest proposal was that of Parker (1955): $\mathcal{E} \propto \overline{\boldsymbol{B}}$. Because $\mathcal{E}$ represented a new way of Generating electric current, he denoted by $\Gamma$ the new constant of proportionality. Subsequently Max Steenbeck, Fritz Krause and Karl-Heinz Rädler, referred to here as 'SKR', used $\alpha$ instead, and their choice has been preferred. Parker's $\Gamma$-effect is now universally called 'the $\alpha$-effect':

$$
\mathcal{E}=\alpha \overline{\boldsymbol{B}}, \quad \text { so that } \eta^{\mathrm{T}} \nabla \times \overline{\boldsymbol{B}}=\overline{\boldsymbol{E}}+\overline{\boldsymbol{U}} \times \overline{\boldsymbol{B}}+\alpha \overline{\boldsymbol{B}} .
$$

Comparing the last 2 terms in $(1.1 b)$, it is seen that $\alpha$ is a pseudoscalar, i.e. a scalar that changes sign on coordinate reflection, $\boldsymbol{x} \rightarrow-\boldsymbol{x}$. After some simplifying assumptions, Steenbeck, Krause \& Rädler (1966) could evaluate $\alpha$ approximately. SKR also proposed and motivated several alternatives to $(1.1 a)$ that are not examined here. (See Krause \& Rädler (1980), Rädler (1966) and other early papers by SKR translated from German by Roberts \& Stix (1971).)

The main significance of the $\alpha$-effect is that it allows $\bar{B}$ to have a persistent self-generated axisymmetric part, despite Cowling's theorem (Cowling 1933). Soon many axisymmetric dynamo models were constructed that made use of that fact, too many models to be referenced here. Parker himself swiftly demonstrated the scientific potential of his idea by using it plausibly to explain the magnetic polarity cycle of the Sun and the progression of solar activity towards the equator during each polarity cycle (Parker 1957).

As Parker (1955) recognized, linear axisymmetric $\alpha$-models succeed because the zonal current $\alpha \bar{B}_{\phi}$, created from the zonal magnetic field $\bar{B}_{\phi}$ by the $\alpha$-effect, produces the meridional magnetic field, $\overline{\boldsymbol{B}}_{\mathrm{M}}$. This in turn creates zonal magnetic field $\bar{B}_{\phi}$ either by the $\alpha$-effect or by zonal shearing of $\overline{\boldsymbol{B}}_{\mathrm{M}}$ (often called 'the $\omega$-effect' and sometimes 'the $\Omega$-effect'), or by both mechanisms working together. At one extreme $\left(|\alpha| \ll r_{o}|\omega|\right)$, where production of $\bar{B}_{\phi}$ by $\alpha$ is negligible and ignored, a regenerative solution is called 'an $\alpha \omega$ dynamo'. Then $(1.1 a)_{\phi}$ is replaced by $r^{2} \boldsymbol{B} \cdot \nabla \omega$. At the other extreme $\left(|\alpha| \gg r_{o}|\omega|\right)$, where the shearing of $\overline{\boldsymbol{B}}_{\mathrm{M}}$ is so ineffective as to be negligible, a regenerative solution is called 'an $\alpha^{2}$ dynamo'. Between the extremes, where $|\alpha|=O\left(r_{o}|\omega|\right)$, both mechanisms create zonal magnetic field, and regenerative models are called $\alpha^{2} \omega$ dynamos; e.g. $\S 2$ of Soward \& Jones (1983). Wu $\&$ Roberts (2014) and this paper provide examples of $\alpha^{2}$ and $\alpha \omega$ dynamos. Many studies, including ours, assume that $\alpha$ and $\omega$ are specified and time independent. A pole-equator temperature difference is often held responsible for creating the zonal shearing from which the $\omega$-effect got its name.

Parker (1955) argued that Coriolis forces would make $\alpha$ non-zero if the turbulence were inhomogeneous as is the case, for example, if the density of the fluid, $\rho(\boldsymbol{x})$, depends on position $\boldsymbol{x}$ through gravitational compression, or through thermal expansion in a temperature-varying environment. Alternatively, Steenbeck et al. (1966) showed that the required inhomogeneity could be due to a gradient in the turbulent intensity, $\sqrt{ }\left(\overline{\boldsymbol{u}^{2}}\right)$, as is appropriate when $\rho$ is nearly uniform and the Boussinesq approximation is adopted, as is done below. Krause (1966) reviewed the ongoing work of the 
Steenbeck-Krause-Rädler team. This paper contained on pages 161-163 a more detailed description of helicity than that of Steenbeck \& Krause (1966) but, as Krause (1966) is a difficult paper to access, we shall refer below to Steenbeck \& Krause (1966). In this they continued the argument that led them to their approximate $\alpha$ (see above), finding that $\alpha$ is proportional to another pseudoscalar $H$, defined from $\boldsymbol{U}$ and the vorticity, $\boldsymbol{\nabla} \times \boldsymbol{U}$, of the flow by

$$
H \equiv \overline{\boldsymbol{U} \cdot(\boldsymbol{\nabla} \times \boldsymbol{U})}
$$

Steenbeck \& Krause (1966) and Krause $(1966, \S 9)$ christened $H$ 'Schraubensinn' ('screwiness') but later Moffatt (1969) proposed the name by which it is now universally known: 'helicity'. Even the translators of Steenbeck \& Krause (1966) chose 'helicity' in preference to 'screwiness'!

As a historical note, we should add that Steenbeck \& Krause (1966) do not average $\boldsymbol{U} \cdot \boldsymbol{\nabla} \times \boldsymbol{U}$ in their $\S 9$. In fact, Braginsky (1965) had already shown that, if the motion $\boldsymbol{U}+\boldsymbol{u}$ were laminar with $\boldsymbol{U}$ being axisymmetric and much greater than the asymmetric $\boldsymbol{u}$, then an $\alpha$-effect $\overline{\mathcal{E}}=\alpha \bar{B}_{\phi} \mathbf{1}_{\phi}$ would be created provided $\boldsymbol{u}$ had finite helicity $H$, the average being over $\phi$ and not over a turbulent ensemble. Braginsky therefore did not need to adopt an ansatz such as (1.1a) because he could evaluate his $\alpha$ explicitly in terms of $\boldsymbol{u}$. Soward (1972) discovered how Braginsky's $\alpha$ is related to $H$; see also Soward \& Roberts (2014). Braginsky (1964) used his $\alpha$ to create kinematic dynamos driven solely by $\boldsymbol{U}+\boldsymbol{u}$, these being the first mean-field models ever constructed that could be said to be mathematically complete.

\subsection{Organization of this paper}

Both spherical coordinates $(r, \theta, \phi)$ and cylindrical coordinates $(s, \phi, z)$ are used below, the north polar axis $O z$ being $\theta=0$ in the former and $s=0$ for $z \geqslant 0$ in the latter. Unit vectors are denoted by a bold unity with a suffix specifying the direction in which the pertinent coordinate increases, e.g. $\mathbf{1}_{\phi}$ and $\mathbf{1}_{z}$ above. The core boundary, $S$, is axisymmetric with respect to the rotation axis $O z$. The northern hemisphere of $S$ is labelled $\mathcal{N}$, and the southern hemisphere $\mathcal{S}$. The interior and the exterior of the core are respectively $\mathrm{v}$ and $\mathrm{v}^{*}$. Ensemble averages are no longer needed below; the overbar will be for averages over $\phi$. For our mean-field models, every scalar field $Q$ and the components of every vector field $\boldsymbol{Q}$ are independent of $\phi$.

We use pre-Maxwell electromagnetic theory and simplify the fluid dynamics by adopting the Boussinesq approximation. We use SI units and, except in $\S \S 1$ and 4.1, we employ the following dimensionless variables: For mass $\mathrm{M}$, length $\mathrm{L}$, time $\mathrm{T}$ and magnetic field $\mathcal{B}$

$$
\mathrm{M} \mapsto \rho r_{o}^{3} \mathrm{M}, \quad \mathrm{L} \mapsto r_{o} \mathrm{~L}, \quad \mathrm{~T} \mapsto\left(\eta / r_{o}^{2}\right) \mathrm{T}, \quad \mathcal{B} \mapsto(2 \Omega \rho \mu \eta)^{1 / 2} \mathcal{B}, \quad(1.2 a-d)
$$

where the density $\rho$ and the magnetic diffusivity $\eta$ are each assumed to be the spatially constant; the angular speed of our reference frame is $\Omega$, and $\mu$ is the magnetic permeability (assumed to be that of free space, $\mu=4 \pi \times 10^{-7} \mathrm{H} \mathrm{m}^{-1}$ ). Also, for velocity, electric current density, $\boldsymbol{J}$, electric field and pressure $\Pi$ (including a part $\left(\rho \Omega^{2} r_{o}^{2}\right) / 2$ from the centrifugal acceleration)

$$
\boldsymbol{U} \mapsto\left(\eta / r_{o}\right) \boldsymbol{U}, \quad \boldsymbol{J} \mapsto\left(2 \Omega \rho \eta / \mu r_{o}^{2}\right)^{1 / 2} \boldsymbol{J}, \quad \boldsymbol{E} \mapsto\left(2 \Omega \rho \mu \eta^{3} / r_{o}^{2}\right)^{1 / 2} \boldsymbol{E}, \quad \Pi \mapsto(2 \Omega \rho \eta) \Pi .
$$


Finally, for kinetic and magnetic energies $\mathcal{K}$ and $\mathcal{M}$, power $\mathcal{P}, \alpha$-effect and specific body force, $\boldsymbol{F}$

$$
\mathcal{K}, \mathcal{M} \mapsto\left(\rho \eta^{2} r_{o}\right)(\mathcal{K}, \mathcal{M}), \quad \mathcal{P} \mapsto\left(\rho \eta^{3} / r_{o}\right) \mathcal{P}, \quad \alpha \mapsto\left(\eta / r_{o}\right) \alpha, \quad \boldsymbol{F} \mapsto\left(2 \Omega \rho \eta / r_{o}\right) \boldsymbol{F}
$$

Our reference frame rotates with the mantle, with angular velocity $\left(2 E_{\eta}\right)^{-1} \mathbf{1}_{z}$, where

$$
E_{\eta} \equiv \eta / 2 \Omega r_{o}^{2}
$$

is the magnetic Ekman number.

This is a sequel to two of our recent papers, Roberts \& Wu (2014) and Wu \& Roberts (2014), which will be referred to respectively as 'paper 1' and 'paper 2'. These are oriented towards the geodynamo but, as here, they ignore the existence of Earth's solid inner core, the core being assumed spherical and entirely fluid. The Earth provides the estimates we make of relevant dimensionless parameters such as $E_{\eta}$. As in paper 2, $\alpha, \boldsymbol{U}$ and $\boldsymbol{B}$ are axisymmetric, i.e. they depend on $s$ and $z$ only; often $Q(s, z)$ is written $Q(\boldsymbol{x})$. Equation $\mathrm{X}, \S \mathrm{Y}$ and figure $\mathrm{Z}$ of paper $\mathrm{n}$ are referred to as $(\mathrm{n}$ : equation $(\mathrm{X})),(\mathrm{n}$ : $\mathrm{Y})$ and $(\mathrm{n}$ : figure $\mathrm{Z})$.

\section{The magnetostrophic approximation}

Magnetostrophy (abbreviated MS) is a term we shall use to mean the absence of viscous friction in Earth's core. This approximation, used throughout this paper, is suggested by the extreme smallness of the ratio of the viscous and Coriolis forces, quantified by the Ekman number $E=v / 2 \Omega r_{o}^{2}$, where $v$ is the core's kinematic viscosity, $\Omega\left(\approx 7.3 \times 10^{-5} \mathrm{~s}^{-1}\right)$ is the angular speed of Earth's rotation and $r_{o}\left(\approx 3.48 \times 10^{6} \mathrm{~m}\right)$ is the radius of the core, assumed spherical. For $v \approx 10^{-6} \mathrm{~m}^{2} \mathrm{~s}^{-1}$, a value widely believed to be realistic for Earth's core, $E \approx 10^{-15}$; e.g. de Wijs et al. (1998), Voĉadlo et al. (2000).

It is generally recognized that core motions are turbulent and, lacking a proper deductive theory of turbulence, several ad hoc representations of turbulent stresses have been made, including ones that simply replace the kinematic viscosity $v$ by a turbulent viscosity, $v_{\text {turb }}$. Although this certainly oversimplifies turbulent dynamics (see e.g. Roberts \& King 2013, §9.2), it has been used to make the estimates $E_{\text {turb }} \approx 6 \times$ $10^{-11}$ (Buffett 1992) and $E_{\text {turb }} \approx 2 \times 10^{-12}$ (Buffett \& Christensen 2007). We believe that these turbulent Ekman numbers are still so small that the MS approximation is justified.

The fully three-dimensional MHD models that today dominate dynamo studies first appeared in the mid-1990s. We shall call them 'today's models'. Their impact has been so great that interest in the MS alternative has waned, even though demands for numerical stability condemn investigators to limit themselves to $E$ vastly greater than the geophysically realistic $10^{-15}$. When $E$ has to exceed $\sim 10^{-6}$ in simulations, Roberts \& King (2013) called them 'viscously dominated'. They pointed out that, even if the Earth's core were made of honey, $E$ would still be less than $10^{-11}$ !

A main preoccupation of dynamo simulation over the past 2 decades has been to reduce the gap between the $E$ of today's models and realistic $E$, using the ever-increasing capability of computer hardware. Recently Schaeffer et al. (2017) were able to reach $E=3 \times 10^{-7}$ in buoyantly driven three-dimensional (3-D) dynamos. Livermore, Jackson \& Hollerbach (2013) reached $E=3 \times 10^{-9}$ in a limited 
simulation that tracked only the toroidal part of the magnetic field. Although it is remarkable what has been achieved in 2 decades, the drive for smaller $E$ becomes increasingly difficult as $E$ is decreased; see Davies, Gubbins \& Jimack (2011). Using Moore's law, Roberts \& King $(2013, \S 9.1)$ estimated that advances in computer technology will be unable before AD 2038 to simulate buoyantly driven 3-D MHD dynamos for $E \approx 10^{-15}$. The ever-increasing difficulty of attaining ever-smaller $E$ is caused principally by the need to resolve numerically the Ekman layers at the core boundaries, whose thicknesses are dimensionally $\delta^{E} \propto E^{1 / 2} r_{o}$. For the numerically attainable $E=10^{-6}, \delta^{E}$ is approximately $3 \mathrm{~km}$; for the unattainable $E=10^{-15}, \delta^{E}$ is about $10 \mathrm{~cm}$. The rate $\mathcal{Q}_{v}$ of energy dissipation through viscosity is (dimensionally) the product of the volume of the boundary layer $O\left(r_{o}^{2} \delta^{E}\right)$ and the dissipation rate per unit volume $O\left(\rho v\left(\mathcal{U} / \delta^{E}\right)^{2}\right)$, where $\mathcal{U}$ is the characteristic speed of core motions; therefore $\mathcal{Q}_{v} \propto 2 \Omega \rho \mathcal{U}^{2} r_{o}^{2} \delta^{E}$. In other words, as $E$ is reduced, the influence of the boundary layers on the mainstream solution (the solution at distances greater than $\delta^{E}$ from boundaries) diminishes until plausibly it is effectively non-existent. The boundary layers themselves become vanishingly thin. Why then continually press today's models to deliver solutions for smaller and smaller $E$ ? Why not follow the example of every other modeller in theoretical physics: if a physical process is unimportant, exclude it from the model?! Although this may motivate magnetostrophic modelling strongly, it is insufficient; more should be said about the limitations of MS.

Today's computer models have one significant attribute lacked by MS: they include the inertial force in full. MS solutions must, however, approximate the inertial force to avoid the large Ekman numbers that make today's computer models irrelevant to the geodynamo and to most cosmic dynamos. This subject is the main topic of $\S 4.1$ below. The MS approximation is often viewed as the logical way to find solutions when viscosity is unimportant, but in reality its success also depends indirectly on the smallness of the inertial force, as measured by the Rossby number, Ro, defined in $\S 3.1$ below.

It is not appropriate here to prolong discussion by including the inner core and the shear layers that plausibly exist at the tangent cylinder, which is the cylinder parallel to $O z$ that touches the inner core at its equator. There is an extensive literature on these shear layers, going back to Proudman (1956) and Stewartson (1966); see Dormy \& Soward (2007). Most MS models, including ours, do not recognize the existence of the inner core, but see Hollerbach (1994) and Livermore \& Hollerbach (2012).

It is natural to hope that today's dynamos for $E \lesssim 10^{-6}$ will differ little from those operating at $E \approx 10^{-15}$, but there are plausible reasons for thinking otherwise. These doubts followed the analysis of Chandrasekhar $(1954,1956)$ of the marginal stability of a rotating Bénard layer in a vertical magnetic field, and were first articulated by Eltayeb \& Roberts (1970) who argued that, when $E$ is small enough, any sufficiently strong magnetic field would facilitate (magneto-)convection so much that magnetic field generation would even be possible at smaller Rayleigh numbers $R a$ than when the magnetic field is smaller or absent, i.e. they postulated two types of buoyantly driven dynamos, a weak-field, viscously dominated branch and a more easily excited, strong-field, magnetically dominated branch. (The Rayleigh number is a non-dimensional measure of the buoyancy force when temperature decreases with increasing height. Chandrasekhar was the first to give it a name. In chapter VI of his celebrated book (Chandrasekhar 1961), he defined $R a$ for a sphere of radius $r_{o}$ uniformly heated by dissolved heat sources. Assuming a constant thermal diffusivity, $\kappa$, the ambient temperature gradient is $\beta=-\beta_{o} r / r_{o}$, where $\beta_{o}$ is the gradient at $r=r_{o}$. 
For uniform fluid density, the gravitational force is $\boldsymbol{g}(\boldsymbol{x})=-g_{o} \boldsymbol{r} / r_{o}$, where $g_{o}$ is gravity at $r=r_{o}$. Chandrasekhar defined the Rayleigh number as $R a=g_{o} \alpha_{T} \beta_{o} r_{o}^{4} / v \kappa$, where $\alpha_{T}$ is the thermal expansion coefficient. For a highly rotating system, particularly in the MS approximation, a more convenient definition is $R a=g_{o} \alpha_{T} \beta_{o} r_{o}^{2} / \Omega \eta$.)

The speculation of Eltayeb \& Roberts (1970) was soon supported by Soward (1974), who derived weak-field solutions for magneto-convection in the Bénard layer for $E \ll 1$. He found that, as the Rayleigh number is increased, these terminated in an asymptote at finite $B$. It was conjectured that this joined to the strong-field branch; see his figure 5. Dormy (2016) recently found that both branches exist when $E=3 \times 10^{-4}$; see his figure 3. Although this encourages further study of MS magneto-convection, our interest below is with mean-field dynamos driven by the $\alpha$-effect alone. There is no obvious physical reason why there should be two branches of $\alpha^{2}$ dynamos, and neither this paper nor papers 1 and 2 indicate that two mean-field branches exist.

Because we ignore viscosity, $\boldsymbol{U}$ can satisfy only one condition on $S$. This is $U_{r}(1, \theta, \phi, t)=0$, which is applied separately on the two hemispheres of $S$ :

$$
U_{r}^{\mathcal{N}}(1, \theta, t)=0, \quad U_{r}^{\mathcal{S}}(1, \theta, t)=0 .
$$

Generally $U_{\theta}(1, \theta, t)$ and $U_{\phi}(1, \theta, t)$ are non-zero.

There is neither viscous coupling between core and mantle in the MS approximation nor magnetic coupling, in the assumed absence of sources of magnetic field in the mantle. We could easily include other coupling mechanisms (topographic, gravitational, viscous, ...; see e.g. Roberts \& Aurnou 2012) but do not do so because we want to avoid complicating further an already complicated message. Therefore $\Gamma_{z}^{\text {total }}$, the $z$-component of the total torque on $\mathrm{v}$, is zero so that the angular momentum, $m_{z}^{\text {total }}$, of core motion about $O z$ cannot change. Our reference frame is the rotating frame in which

$$
m_{z}^{\text {total }} \equiv \int_{\mathrm{v}} s U_{\phi} \mathrm{d}^{3} x=0
$$

We start our numerical integrations from the state

$$
\boldsymbol{B}(\boldsymbol{x}, 0)=B_{0}\left[3 s z \mathbf{1}_{s}+\left(5-6 s^{2}-3 z^{2}\right) \mathbf{1}_{z}\right], \quad \boldsymbol{U}(\boldsymbol{x}, 0)=\mathbf{0},
$$

where $B_{0}$ is an arbitrary constant representing magnetic field strength. This magnetic field is purely dipolar and is created by a zonal current density $J_{\phi}$ proportional to $s$, so that the Lorentz force is $\boldsymbol{J} \times \boldsymbol{B}=\nabla\left[(1 / 2)\left(3 B_{0} s\right)^{2}\left(5-3 r^{2}\right)\right]$. It is therefore in hydrostatic balance with constant $\Pi+(1 / 2)\left(3 B_{0} s\right)^{2}\left(5-3 r^{2}\right)$, making it what in $\S 3.1$ will be called a 'Taylor state'. Clearly $(2.1 a, b)$ are satisfied by $(2.3 b)$, which also implies $m_{z}^{\text {total }}=0$.

A short digression on equatorial symmetry may be welcome here. Although zero in $(2.3 a)$, a non-zero $B_{\phi}(s, z, t)$ is generated in the evolution of $\boldsymbol{B}$ and $\boldsymbol{U}$ that we study below. This, like $B_{s}(s, z, t)$, is antisymmetric in $z$ : i.e. $B_{\phi}(s,-z, t) \equiv-B_{\phi}(s, z, t)$. The antisymmetry of $B_{s}$ and $B_{\phi}$ is preserved as $\boldsymbol{B}$ evolves, as is the $z$-symmetry of $B_{z}: B_{z}(s,-z, t) \equiv B_{z}(s, z, t)$. This type of $z$-symmetry is termed 'dipole symmetry'. Also, starting from $(2.3 b), \boldsymbol{U}$ becomes non-zero and quadrupolar, with $z$-symmetric $s$ and $\phi$-components and a $z$-antisymmetric $z$-component. In addition to this family of solutions in which $\boldsymbol{B}$ is permanently dipolar and $\boldsymbol{U}$ permanently quadrupolar, there is another family in which both $\boldsymbol{B}$ and $\boldsymbol{U}$ are permanently quadrupolar. Neither that family nor mixed dipolar/quadrupolar states are considered here. 
The adjectives geostrophic and ageostrophic often appear in papers $1 \& 2$. There, as in almost all theoretical work in this area including this paper, the system is homogeneous and $\mathrm{v}$ is axisymmetric with respect to the rotation axis. (The axisymmetry of $\boldsymbol{B}$, assumed in paper 2 and assumed in most mean-field models results in further simplification.) Axisymmetry of $\mathrm{v}$ simplifies the definition of geostrophy; see Greenspan (1968). The geostrophic cylinder $\mathcal{C}(s)$ of radius $s$ is defined by $0 \leqslant \phi<2 \pi$ and by $-z_{1}(s) \leqslant z \leqslant z_{1}(s)$ where $z_{1}(s)=\sqrt{ }\left(1-s^{2}\right)$. Its area is $\mathcal{A}(s)=4 \pi s z_{1}(s)$. The cylinder $\mathcal{C}(s)$ is part of the boundary of a volume $\mathcal{V}(s)$ consisting of the interior of $\mathrm{v}$ together with northern and southern extensions bounded by spherical caps $\mathcal{N}(s)$ and $\mathcal{S}(s)$ which are segments of $S$ that complete the boundary of $\mathcal{V}(s)$. The geostrophic average of a scalar function $Q(\boldsymbol{x})$ is

$$
Q^{\mathrm{G}}(s)=\frac{1}{2 z_{1}(s)} \int_{-z_{1}(s)}^{z_{1}(s)} Q(s, z) \mathrm{d} z .
$$

As $s \rightarrow 1, Q(s, z) \rightarrow Q(1,0)$ so that $Q^{\mathrm{G}}(s) \rightarrow Q(1,0)$. If $Q(s, z) \propto z^{2}$ as $s \rightarrow 1$, then $Q^{\mathrm{G}}(s) \propto z_{1}^{2}$, etc.

The ageostrophic (or non-geostrophic) part of $Q(\boldsymbol{x})$ is what remains after the geostrophic part $Q^{\mathrm{G}}(s)$ has been removed:

$$
Q^{\mathrm{A}}(\boldsymbol{x})=Q(\boldsymbol{x})-Q^{\mathrm{G}}(s) .
$$

The geostrophic and ageostrophic parts of a vector such as $\boldsymbol{U}(\boldsymbol{x}, t)$ singles out its $\phi$ component:

$$
\boldsymbol{Q}^{\mathrm{A}}(\boldsymbol{x})=\boldsymbol{Q}(\boldsymbol{x})-\boldsymbol{Q}^{\mathrm{G}}(s)=\boldsymbol{Q}(s, z)-Q_{\phi}^{\mathrm{G}}(s) \mathbf{1}_{\phi},
$$

so that, for example,

$$
U_{s, z}^{\mathrm{A}}(\boldsymbol{x}, t)=U_{s, z}(s, z, t), \quad U_{\phi}^{\mathrm{A}}(\boldsymbol{x}, t)=U_{\phi}(s, z, t)-U_{\phi}^{\mathrm{G}}(s, t), \quad\left[U_{\phi}^{\mathrm{A}}(\boldsymbol{x}, t)\right]^{\mathrm{G}}(s, t)=0 .
$$

\section{Basic equations and boundary conditions}

Finding the magnetic field $\boldsymbol{B}$ created by the fluid velocity $\boldsymbol{U}$ is a standard kinematic dynamo problem that has been so often solved that we shall not dwell on it here. We shall merely, for reference purposes, state that when the $\alpha$-effect is included, $\boldsymbol{B}$ and $\boldsymbol{E}$ satisfy the pre-Maxwell equations. In $\mathrm{v}$

$$
\boldsymbol{J}=\nabla \times \boldsymbol{B}, \quad \nabla \times \boldsymbol{E}=-\partial_{t} \boldsymbol{B}, \quad \nabla \cdot \boldsymbol{B}=0, \quad \boldsymbol{J}=\boldsymbol{E}+\boldsymbol{U} \times \boldsymbol{B}+\alpha \boldsymbol{B} . \quad(3.1 a-d)
$$

The induction equation that follows from $(3.1 a-d)$ is

$$
\frac{\partial \boldsymbol{B}}{\partial t}=\nabla \times(\boldsymbol{U} \times \boldsymbol{B}+\alpha \boldsymbol{B})+\nabla^{2} \boldsymbol{B} .
$$

In the electrically insulating exterior $\mathrm{v}^{*}$ of $\mathrm{v},(3.1 a-c)$ hold but $(3.1 d)$ is replaced by $\boldsymbol{J}^{*} \equiv \mathbf{0}$.

The solution to (3.1e) has to obey the continuity condition,

$$
\boldsymbol{B}=\boldsymbol{B}^{*} \quad \text { on } r=1, \quad \text { and } \quad r^{2} \boldsymbol{B}^{*} \rightarrow \mathbf{0} \quad \text { as } r \rightarrow \infty,
$$


the latter excluding sources of $\boldsymbol{B}$ at infinity. Equations $(3.1 f, g)$ lead to the so-called 'dynamo conditions', which for axisymmetric $\boldsymbol{B}$, are

$$
B_{\phi}(1, \theta, t)=0, \quad\left[\frac{\partial B_{r, n}}{\partial r}+(n+2) B_{r, n}\right]_{(1, t)}=0 \quad \forall n \geqslant 0
$$

where $B_{r, n}(r, t)$ is the $n$th spherical harmonic components of $B_{r}(\boldsymbol{x}, t)$.

In $\S 4$, the geostrophic and ageostrophic parts of the induction $(3.1 e)$ will be needed. These are defined by

$$
\begin{gathered}
\partial_{t} \boldsymbol{B}(\boldsymbol{x}, t)=\boldsymbol{C}^{\mathrm{G}}(\boldsymbol{x}, t)+\boldsymbol{C}^{\mathrm{A}}(\boldsymbol{x}, t), \quad \text { where } \\
\boldsymbol{C}^{\mathrm{G}}(\boldsymbol{x}, t)=\nabla \times\left(\boldsymbol{U}^{\mathrm{G}} \times \boldsymbol{B}\right), \quad \boldsymbol{C}^{\mathrm{A}}(\boldsymbol{x}, t)=\nabla \times\left(\boldsymbol{U}^{\mathrm{A}} \times \boldsymbol{B}+\alpha \boldsymbol{B}\right)+\nabla^{2} \boldsymbol{B} .
\end{gathered}
$$

In the axisymmetric case, it was shown in (2: equation (18d)) that

$$
\boldsymbol{C}^{\mathrm{G}}(\boldsymbol{x}, t)=s Z^{\mathrm{G}}(s, t) B_{s}(\boldsymbol{x}, t) \mathbf{1}_{\phi}, \quad \text { where } Z^{\mathrm{G}}(s, t)=\frac{\partial \zeta(s, t)}{\partial s} \text { and } \zeta(s, t)=\frac{U_{\phi}^{\mathrm{G}}(s, t)}{s}
$$

is the geostrophic shear.

In MS theory, the Boussinesq equations governing $\boldsymbol{U}(\boldsymbol{x}, t)$ in mean-field models satisfies

$$
E_{\eta} D_{t} \boldsymbol{U}+\mathbf{1}_{z} \times \boldsymbol{U}=-\boldsymbol{\nabla} \Pi+\boldsymbol{F}, \quad \boldsymbol{\nabla} \cdot \boldsymbol{U}=0, \quad \text { where } \boldsymbol{F}=\boldsymbol{J} \times \boldsymbol{B} \quad(3.3 a-c)
$$

is the Lorentz force, which provides the power that maintains $\boldsymbol{U}(\boldsymbol{x}, t)$ and, when there is dynamo action, maintains $\boldsymbol{B}(\boldsymbol{x}, t)$ too; in $(3.3 a), D_{t}\left(=\partial_{t}+\boldsymbol{U} \cdot\right)$ is the material derivative. For simplicity, the possibility that $\boldsymbol{F}$ contains a radial buoyancy force has been excluded in paper 2 and in this paper $(\$ 2)$. The $\alpha$-effect alone powers our mean-field $\alpha^{2}$ dynamos, but our $\alpha \omega$ models are powered too by the rate of working of the imposed shearing velocity against the Lorentz force; see below.

Mass conservation (3.3b) and the boundary conditions $(2.1 a, b)$ imply (Taylor 1963)

$$
U_{s}^{\mathrm{G}}(s, t)=0 \text { for all } 0 \leqslant s \leqslant 1 .
$$

Apart from the pressure gradient, only the Lorentz and Coriolis forces affect the motion of fluid in the volume $\mathcal{V}(s)$ defined in $\S 2$. Its equation of motion is, by $(3.3 a)$

$$
E_{\eta}\left[\partial_{t} m_{z}(s, t)+f_{s}(s, t)\right]=\Gamma_{z}(s, t)
$$

where $m_{z}(s, t)$ is the $z$-component of the angular momentum of $\mathcal{V}(s)$,

$$
m_{z}(s, t) \equiv \mathbf{1}_{z} \cdot \int_{\mathcal{V}(s)} \boldsymbol{x} \times \boldsymbol{U} \mathrm{d}^{3} x=\int_{0}^{s} s\left[\int_{\mathcal{C}(s)} U_{\phi}(\boldsymbol{x}, t) \mathrm{d}^{2} x\right] \mathrm{d} s=\int_{0}^{s} s \mathcal{A}(s) U_{\phi}^{\mathrm{G}}(s, t) \mathrm{d} s,
$$


$f_{s}(s, t)$ is the flux of angular momentum from $\mathcal{V}(s)$ across $\mathcal{C}(s)$,

$$
f_{s}(s, t)=\int_{\mathcal{C}(s)} s U_{s}(\boldsymbol{x}, t) U_{\phi}(\boldsymbol{x}, t) \mathrm{d}^{2} x=s \mathcal{A}(s)\left(U_{s} U_{\phi}\right)^{\mathrm{G}}(s, t),
$$

and $\Gamma_{z}(s, t)$ is the $z$-component of the torque on $\mathcal{V}(s)$,

$$
\Gamma_{z}(s, t) \equiv \mathbf{1}_{z} \cdot \int_{\mathcal{V}(s)} \boldsymbol{x} \times \boldsymbol{F} \mathrm{d}^{3} x=\int_{0}^{s} s\left[\int_{\mathcal{C}(s)} F_{\phi}(\boldsymbol{x}, t) \mathrm{d}^{2} x\right] \mathrm{d} s=\int_{0}^{s} s \mathcal{A}(s) F_{\phi}^{G}(s, t) \mathrm{d} s .
$$

By writing the Lorentz force (3.3c) as the divergence of the magnetic stress tensor, and applying the dynamo condition $(3.1 h), \Gamma_{z}(s, t)$ can be expressed as a surface integral:

$$
\Gamma_{z}(s, t)=E_{\eta}^{-1} \int_{\mathcal{C}(s)} s B_{s} B_{\phi} \mathrm{d}^{2} x
$$

Differentiating $(3.5 a)$ by $t$, applying $(3.2 a, d)$ and adding a star to Taylor's $\alpha$ to avoid confusion with the $\alpha$-effect, we find

$$
\begin{gathered}
E_{\eta} \frac{\partial \Gamma_{z}(s, t)}{\partial t}=s\left[s \alpha^{*} Z(s, t)-S(s, t)\right], \quad \text { where } \alpha^{*}(s, t)=\int_{\mathcal{C}(s)} B_{s}^{2} \mathrm{~d}^{2} x \quad \text { and } \\
S(s, t)=-\int_{\mathcal{C}(s)}\left(B_{s} C_{\phi}^{\mathrm{A}}+B_{\phi} C_{s}^{\mathrm{A}}\right) \mathrm{d}^{2} x .
\end{gathered}
$$

Equation (3.5b) determines $Z(s, t)$ from $\Gamma_{z}(s, t), S(s, t)$ and $\alpha^{*}(s, t)$. For dipolar $\boldsymbol{B}$, these are each $O\left(s^{4}\right)$ as $s \rightarrow 0$; for quadrupolar $\boldsymbol{B}$, they are each $O\left(s^{2}\right)$ as $s \rightarrow 0$. In both these cases, $Z(s, t)=O\left(s^{-1}\right), \zeta(s, t)=O(\log s)$ and $U_{\phi}^{\mathrm{G}}(s, t)=O(s \log s)$ for $s \rightarrow 0$. This weak singularity of $U_{\phi}^{\mathrm{G}}(s, t)$ may be an endemic shortcoming of axisymmetric MS models and of the axisymmetric part of more general, three-dimensional MS models.

By $(3.1 h),(3.4 d),(3.5 a)$, and $B_{\phi}(1, t)=0$, by

$$
\Gamma_{z}(1, t)\left(=\Gamma_{z}^{\mathrm{total}}\right)=0, \quad \partial_{s} \Gamma_{z}(1, t)=0, \quad F_{\phi}^{\mathrm{G}}(1, t)=0 .
$$

Finally, to show that $\alpha$ is the energy source driving the $\alpha^{2}$ system, we develop the energy equation. The total kinetic and magnetic energies are

$$
\mathcal{K}=\frac{1}{2} \int_{\mathrm{v}} \boldsymbol{U}^{2} \mathrm{~d}^{3} x, \quad \mathcal{M}=\frac{1}{2} E_{\eta}^{-1} \int_{\mathrm{v}_{\infty}} \boldsymbol{B}^{2} \mathrm{~d}^{3} x
$$

where $\mathrm{v}_{\infty}=\mathrm{v}+\mathrm{v}^{*}$ is all space. By taking the scalar product of $(3.3 a)$ with $\boldsymbol{U}$ and the scalar product of $(3.1 e)$ with $\boldsymbol{B}$, integrating over $\mathrm{v}$ and applying the associated boundary conditions, it is found that

$$
E_{\eta} \frac{\partial \mathcal{K}}{\partial t}=\mathcal{L}, \quad \frac{\partial \mathcal{M}}{\partial t}=\mathcal{P}-\mathcal{L}-\mathcal{Q}
$$


where $\mathcal{L}$ is the rate of working of the Lorentz force, $\mathcal{P}$ is the power input and $\mathcal{Q}(t)$ is the dissipation, which is entirely ohmic in the MS approximation:

$$
\mathcal{L}=\int_{\mathrm{v}} \boldsymbol{U} \cdot(\boldsymbol{J} \times \boldsymbol{B}) \mathrm{d}^{3} x, \quad \mathcal{P}=\int_{\mathrm{v}} \alpha \boldsymbol{J} \cdot \boldsymbol{B} \mathrm{d}^{3} x, \quad \mathcal{Q}=\int_{\mathrm{v}} J^{2} \mathrm{~d}^{3} x, \quad(3.6 e-g)
$$

Clearly $\partial_{t}\left(E_{\eta} \mathcal{K}+\mathcal{M}\right)=\mathcal{P}-\mathcal{Q}$. As stated above, the power input, $\mathcal{P}$, is created by $\alpha$. Because the integrand of (3.6f) is the product of two helicities, the kinetic helicity $\alpha$ and 'the current helicity' $\boldsymbol{J} \cdot \boldsymbol{B}$, which is a pseudoscalar too, $\mathcal{P}$ is correctly independent of the right- or left-handedness of the coordinate frame.

In a steadily operating $\alpha^{2}$ dynamo, $(3.6 c, d)$ show that $\langle\mathcal{L}\rangle=0$ and $\langle\mathcal{P}\rangle=\langle\mathcal{Q}\rangle$, where $\langle Q\rangle$ is the average of $Q(t)$ over a sufficiently long time, e.g. the magnetic diffusion time, which is $O(1)$ in our non-dimensionalization. The positivity of $\mathcal{P}$ implies that, in a steadily working $\alpha^{2}$ dynamo, the kinetic helicity on average creates a current helicity of the same sign.

The kinetic energy $\mathcal{K}$ in (3.6a) may be written as $\mathcal{K}=(1 / 2) \int_{\mathrm{v}}\left[U_{\mathrm{M}}^{2}+s^{2} \zeta^{2}\right] \mathrm{d}^{3} x$ where $\boldsymbol{U}_{\mathrm{M}}=U_{s} \mathbf{1}_{s}+U_{z} \mathbf{1}_{z}$ is the meridional part of $\boldsymbol{U}$. For an $\alpha^{2} \omega$ dynamo, defined in $\S 1$, this is replaced by $\mathcal{K}=(1 / 2) \int_{\mathrm{v}}\left[U_{\mathrm{M}}^{2}+s^{2}(\zeta+\omega)^{2}\right] \mathrm{d}^{3} x$ which, in the extreme case of an $\alpha \omega$ dynamo $\left(|\alpha| \ll r_{o}|\omega|\right)$, is $\mathcal{K}=(1 / 2) \int_{\mathrm{v}}\left[U_{\mathrm{M}}^{2}+s^{2} \omega^{2}\right] \mathrm{d}^{3} x$.

\subsection{Original Taylor theory (OTT); Taylor's constraint}

This subsection summarizes what is needed later in this paper about OTT, our abbreviation for 'original Taylor theory' (Taylor 1963).

The study of magnetostrophy was initiated by the plasma physicist Bryan Taylor, whose paper had a major impact on the dynamo community. As well as neglecting viscosity, Taylor (1963) discarded the inertial force in the rotating frame. Inertia is generally quantified by the Rossby number $R o \equiv \mathcal{U} / 2 \Omega r_{o}$. For an estimated flow speed $\mathcal{U}=2 \times 10^{-4} \mathrm{~m} \mathrm{~s}^{-1}, R o \approx 4 \times 10^{-7}$. Our choice of non-dimensional variables makes it more convenient to use instead the magnetic Ekman number (1.5). For $\eta \approx 0.7 \mathrm{~m}^{2} \mathrm{~s}^{-1}$ (Pozzo et al. 2012), $E_{\eta} \approx 4 \times 10^{-10}$. The ratio of these 2 inertial measures is the magnetic Reynolds number: $R m \equiv \mathcal{U} r_{o} / \eta=R o / E_{\eta}$, which is approximately 1000 . The omission of the inertial force may therefore be stated either as $R o=0$ or as $E_{\eta}=0$. In writing $E_{\eta}=0$, there is no implication that $\eta$ is as negligible as $v ; E_{\eta}$ is the ratio of the dynamo time scale, $\tau_{\eta}=r_{o}^{2} / \eta$, and the other time scales governing geodynamo (identified in $\S 4.1$ below) and is much greater than any of them; $E_{\eta}=0$ is an expression of that fact.

The smallness of $R o$ encouraged Taylor (1963) to ignore inertia entirely in the rotating frame. For $E_{\eta}=0$, the Boussinesq MS equations $(3.3 a, b)$ governing $\boldsymbol{U}$ become

$$
\mathbf{1}_{z} \times \boldsymbol{U}=-\nabla \Pi+\boldsymbol{F}, \quad \boldsymbol{\nabla} \cdot \boldsymbol{U}=0 .
$$

The derivation of energy conservation given in $\S 3$ remains valid for $\mathcal{K}=0$. The equation of motion $(3.4 a)$ of $\mathcal{V}(s)$ and its $s$-differential are also simplified, becoming

$$
\Gamma_{z}(s, t) \equiv 0, \quad F_{\phi}^{\mathrm{G}}(s, t)=0, \quad \text { for all } 0 \leqslant s \leqslant 1 \text { and all } t .
$$

Equivalent to $(3.7 d)$ is

$$
\int_{\mathcal{C}(s)}(\boldsymbol{J} \times \boldsymbol{B})_{\phi} \mathrm{d}^{2} x=0, \quad \text { for all } 0 \leqslant s \leqslant 1 \text { and all } t .
$$


Either of $(3.7 d, e)$, but usually (3.7e), is termed 'Taylor's constraint' (aka 'Taylor's condition'). Provided it is satisfied, the solution of $(3.7 a, b)$ can almost be reduced to quadratures, as Taylor (1963) demonstrated.

To maintain his constraint, Taylor (1963, equation (4.5)) derived a second-order equation for $U_{\phi}^{\mathrm{G}}(s, t)$. The first integral of that equation is

$$
Z(s, t)=\frac{q(s, t)}{s}, \quad \text { where } q(s, t)=\frac{S(s, t)}{\alpha^{*}(s, t)} .
$$

The function $q(s, t)$ defined by $(3.8 b)$ is regular for $0 \leqslant s \leqslant 1$ and non-zero at $s=$ 0 , as shown below $(3.5 d)$. This means that $\zeta(s, t)$ cannot be obtained by integrating $Z(s, t)$ directly because of the logarithmic divergence found at $s=0$ in $\S 3$. Instead we introduce $\epsilon(>0)$ and define $\zeta^{\epsilon}(s, t)$ by excluding $s=0$ from the range of integration, using (3.8a) to obtain

$$
\zeta^{\epsilon}(s, t)=\int_{\epsilon}^{s} \frac{S(s, t)}{s \alpha^{*}(s, t)} \mathrm{d} s+D_{0}(t), \quad \text { so that } \zeta^{\epsilon}(s, t) \sim q(0, t) \ln \left(\frac{s}{\epsilon}\right)+D_{0}(t) \text { for } s \rightarrow 0,
$$

where $D_{0}(t)$ is the integration 'constant'. Clearly $\zeta^{\epsilon}(s, t)$ is finite, but diverges as $s \rightarrow 0$ or $\epsilon \rightarrow 0$. We approximated $\zeta(s, t)$ numerically by

$$
\zeta(s, t) \approx \zeta^{\epsilon}(s, t), \quad \text { where } \epsilon=s_{1}
$$

is the smallest positive grid point (at $s=0$, we arbitrarily take $\zeta^{\epsilon}(0, t)=0$ ). Equation (2.2) shows that

$$
D_{0}(t)=-\frac{15}{4 \pi} \int_{0}^{1} s^{2} z_{1} \zeta(s, t) \mathrm{d}^{2} x
$$

(Even though this result was derived from (3.8c), it features $\zeta$ and not $\zeta^{\epsilon}$. This is because $\lim _{\epsilon \rightarrow 0} m_{z}^{\epsilon \text {,total }}=m_{z}^{\text {total }}$.)

Most modellers, including Taylor (1963) and ourselves in this paper, assume the core is fluid throughout, ignoring the inner core entirely. Livermore, Ierley \& Jackson (2009) provide general examples of full-sphere Taylor states. Recognition of the inner core introduces complications touched on in $\S 2$ above. Nevertheless, the behaviour of solutions as $s \rightarrow 0$ is not much affected; the logarithmic divergence of $\zeta$ persists as does the endemic shortcoming of $U_{\phi}^{\mathrm{G}}(s, t)$ identified in $\S 3$.

In the present axisymmetric case, the cylindrical components of (3.7a) are

$$
U_{s}(\boldsymbol{x}, t)=F_{\phi}(\boldsymbol{x}, t), \quad U_{\phi}(\boldsymbol{x}, t)=-F_{s}(\boldsymbol{x}, t)+\partial_{s} \Pi(\boldsymbol{x}, t), \quad U_{z}(\boldsymbol{x}, t)=-s^{-1} \partial_{s}\left(s z_{1} \widetilde{F}_{\phi}\right),
$$

where

$$
\begin{gathered}
\Pi(\boldsymbol{x}, t)=z_{1} \widetilde{F}_{z}(\boldsymbol{x}, t)+w(s, t), \quad \frac{\partial w(s, t)}{\partial s}=F_{s}^{\mathrm{G}}(s, t)+\frac{1}{z_{1}} \frac{\partial}{\partial s}\left(s\left[z F_{z}\right]^{\mathrm{G}}(s, t)\right), \\
\widetilde{Q}(\boldsymbol{x}, t)=\frac{1}{2 z_{1}}\left(\int_{z_{1}}^{z} Q(s, z, t)+\int_{-z_{1}}^{z} Q(s, z, t)\right) .
\end{gathered}
$$


Solutions of these equations were given in (1: $\S 4)$, where we recognized that the initial $\boldsymbol{B}(\boldsymbol{x}, 0)$, given for example by $(2.1 a)$, determines the initial $\boldsymbol{U}_{\phi}^{\mathrm{G}}(s, 0)$.

Finding OTT solutions promises to be much easier than solving (3.1e) and the unapproximated partial differential equations governing $\boldsymbol{U}$ that include viscous and inertial forces. This explains why Taylor's paper made a major impact on the dynamo community with its publication in 1963. Nevertheless, the task proved to be more onerous than it appeared to be at first sight, and it took 52 years for Taylor's idea to be successfully implemented. See $(2: \S \S 5,6)$, which presents solutions for mean-field $\alpha^{2}$ and $\alpha \omega$-dynamos.

\section{Modified Taylor theory (MTT); torsional waves}

In this section, a generalization of OTT will be described which, as in paper 1, is termed 'modified Taylor theory' (abbreviated as 'MTT'). Numerical consequences are presented in $\S 5.3$ below. MTT recognizes the existence of torsional waves which OTT does not. Much has been written about torsional waves since their importance was first recognized by Braginsky (1970). See, for example, Wicht \& Christensen (2010), Teed, Jones \& Tobias (2013, 2015), Schaeffer et al. (2017). Interpretations of observations that appear to confirm that torsional waves are travelling across the core are due to Abarca del Rio, Gambis \& Salstein (2000), Gillet et al. (2010) and Holme \& De Viron (2013), who link torsional oscillations to observed changes in the length of day. But we should emphasize here, however, that the incorporation of torsional waves into our MS dynamo model is not our main objective. We have studied MTT primarily because it not only generalizes OTT but also because it is, as we see it, the best MS approximation possible, without re-introducing the shortcomings inherent in today's viscously dominated models. We defend that assertion in $\S 4.1$ below.

MTT ignores the nonlinearity of the inertial force; the $f_{s}$ defined by (3.4c) is now zero, so that $(3.4 a, b, d)$ and $(3.3 d)$ imply

$$
E_{\eta} \frac{\partial U_{\phi}^{\mathrm{G}}(s, t)}{\partial t}=\frac{E_{\eta}}{s \mathcal{A}(s)} \frac{\partial^{2} m_{z}(s, t)}{\partial s \partial t}=\frac{1}{s \mathcal{A}(s)} \frac{\partial \Gamma_{z}(s, t)}{\partial s}=F_{\phi}^{\mathrm{G}}(s, t) .
$$

The left-hand side of (4.1) is the only part of the inertial force $E_{\eta}\left(\partial_{t} \boldsymbol{U}+\boldsymbol{U} \cdot \boldsymbol{\nabla} \boldsymbol{U}\right)$ retained in MTT; the momentum equations $(3.3 a-c)$ and $(3.7 a-c)$ are replaced by

$$
E_{\eta} \partial_{t} U_{\phi}^{\mathrm{G}} \mathbf{1}_{\phi}+\mathbf{1}_{z} \times \boldsymbol{U}=-\nabla \Pi+\boldsymbol{F}, \quad \boldsymbol{\nabla} \cdot \boldsymbol{U}=0, \quad \boldsymbol{F}=\boldsymbol{J} \times \boldsymbol{B} . \quad(4.2 a-c)
$$

The derivation of energy conservation given in $\S 3$ continues to be valid provided the kinetic energy $\mathcal{K}$ in $(3.6 a)$ is redefined with $U_{\phi}^{\mathrm{G}}(s, t)$ replacing $\boldsymbol{U}(\boldsymbol{x}, t)$.

Although $(3.3 d)$ remains true $\left(U_{s}^{\mathrm{G}}(s, t)=0\right),(4.2 a-c)$ no longer imply Taylor's constraint $(3.7 d, e)$. Because the predictive (4.1) replaces the diagnostic (3.7a), it does not constrain $\boldsymbol{F}$. Instead

$$
E_{\eta} \frac{\partial U_{\phi}^{\mathrm{G}}(s, t)}{\partial t}=\frac{1}{\mathcal{A}(s)} \int_{\mathcal{C}(s)}(\boldsymbol{J} \times \boldsymbol{B})_{\phi} \mathrm{d}^{2} x, \quad \text { for all } 0 \leqslant s \leqslant 1 .
$$

When integrating $(4.2 d)$ from an initial state such as $(2.3 a, b)$, the resulting $U_{\phi}^{\mathrm{G}}(s, t)$ evidently depends on $E_{\eta}$, and (4.1) shows that

$$
\partial_{t}\left(E_{\eta} s \mathcal{A}(s) U_{\phi}^{\mathrm{G}}(s, t)\right)=\partial_{s} \Gamma_{z} .
$$


(a)

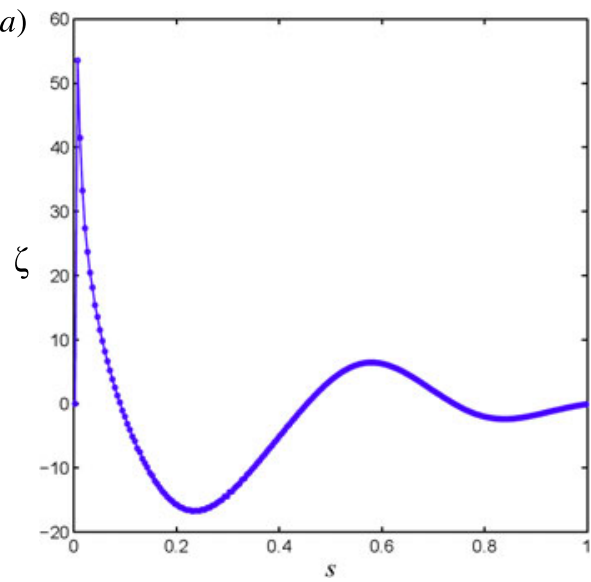

(b)

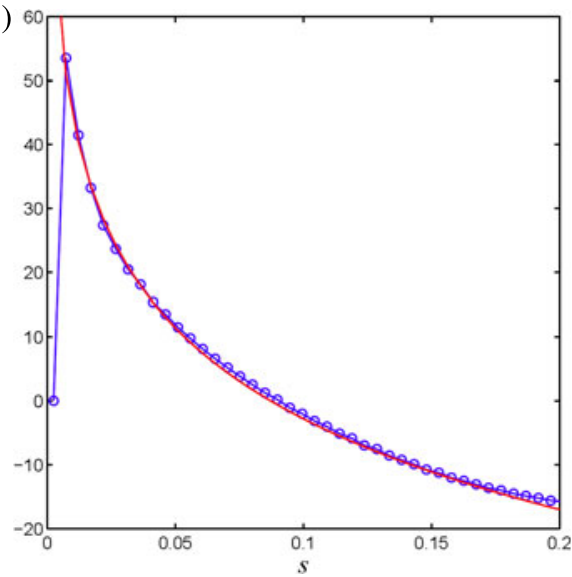

FIgURE 1. The function $\zeta(s, t)$ at $t=54.8$ as a function of $s$ for the modified Taylor theory $\alpha^{2}$ model with $\alpha_{0}=14.5, E_{\eta}=0.01$ and $U_{\phi}^{\mathrm{G}}(s, 0)=0$ is shown with blue curves marked by circles: $(a)$ in the range $0<s \leqslant 1$, and $(b)$ in $0<s \leqslant 0.2$. (The function $U_{\phi}^{\mathrm{G}}(s, t)$ at the same time is shown in figure 3 below.) Also shown in red in $(b)$ is the curve $\zeta(s, t)=-20.5 \ln (s)-50$.

By writing (4.2d) in this conservative form, the condition (2.2) on the total angular momentum $\left(m_{z}^{\text {total }}(t)=0\right)$, is automatically satisfied as $\boldsymbol{U}(\boldsymbol{x}, t)$ evolves (provided that $U_{\phi}^{\mathrm{G}}(1,0)=0$ so that $m_{z}^{\text {total }}$ is initially zero). Equations $(3.5 e)$ and $(4.2 d)$ imply

$$
\partial_{t} U_{\phi}^{\mathrm{G}}(1, t)=0, \quad \text { so that } U_{\phi}^{\mathrm{G}}(1, t)=U_{\phi}^{\mathrm{G}}(1,0)=\text { const. }=K, \text { say. }
$$

In MTT, as for OTT, $\zeta(s, t)$ is $O(\ln s)$ for $s \rightarrow 0$. See figure 1 , which was derived by integrating (4.3a). Except for the first half-grid point, the computed values of $\zeta$ fit well onto the curve $\zeta\left(s, t_{0}\right)=-20.5 \ln s-50$ shown, where $t_{0}=54.8$ is the time at which the data were taken.

In OTT, $(3.4 a)$ is the tautology $0=0$; its left-hand side vanishes because $E_{\eta}=$ 0 and its right-hand side because of Taylor's constraint $(3.7 d, e)$. An important part of Taylor's paper $(1963, \S 4)$ is devoted to finding his $u_{\phi}(s, t)$, i.e. our $U_{\phi}^{\mathrm{G}}(s, t)$. We used his method in deriving OTT results in paper 2 but cannot apply it here because $F_{\phi}^{\mathrm{G}}(s, t)$ is generally non-zero in MTT.

In $\operatorname{MTT}, \Gamma_{z}(s, t)$ and $m_{z}(s, t)$ are generally non-zero and are related by $(3.4 a)$ with $f_{s}(s, t)=0$. They are responsible for coupling geostrophic cylinders together and for time variation of the angular momentum, both of which are associated with torsional waves and both of which are lacking in OTT. An equation governing $\zeta(s, t)\left(=U_{\phi}^{\mathrm{G}}(s, t) / s\right)$ alone can by found by differentiating $(3.5 b)$ by $s$ and then applying $(4.2 d)$, to give

$$
E_{\eta} s^{2} \mathcal{A}(s) \frac{\partial^{2} \zeta}{\partial t^{2}}=\frac{\partial}{\partial s}\left[s\left(s \alpha^{*}(s, t) \frac{\partial \zeta(s, t)}{\partial s}-S(s, t)\right)\right] .
$$

Equation (4.4a) governs a one-dimensional type of Alfvén wave that travels in the $\pm s$ direction. This torsional wave equation may be re-written as (see (1: equation (22a)))

$$
\frac{\partial^{2} \zeta}{\partial t^{2}}=\frac{1}{s^{2} \mathcal{A}} \frac{\partial}{\partial s}\left(s^{2} \mathcal{A} \mathcal{V}_{\text {tort }}^{2} \frac{\partial \zeta}{\partial s}\right)-\frac{1}{s^{2} \mathcal{A} E_{\eta}} \frac{\partial(s S)}{\partial s},
$$




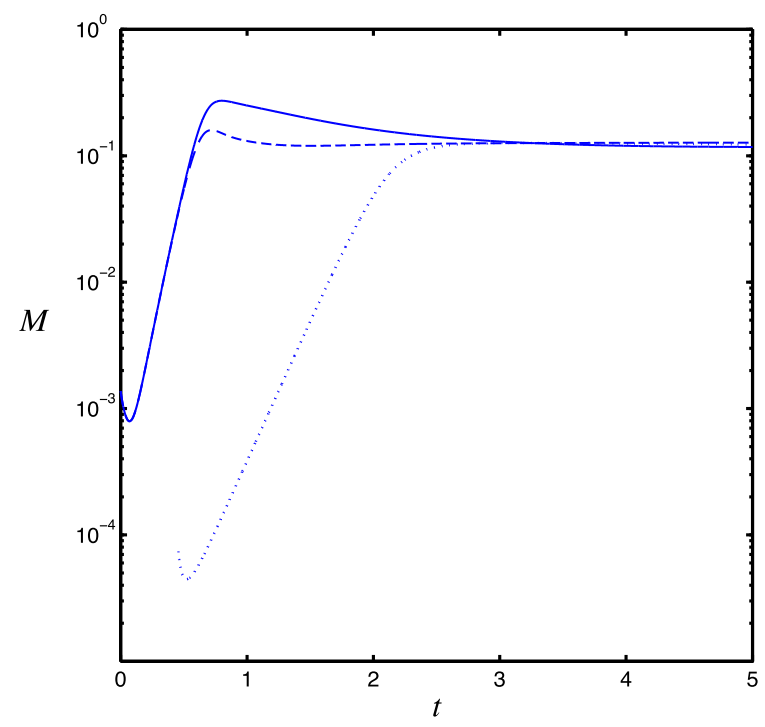

FIGURE 2. Internal magnetic energy, $M(t)$, as a function of time for the $\alpha^{2}$ model for $\alpha_{0}=14.5$. The solid curve is for MTT with $U_{\phi}^{\mathrm{G}}(1,0)=0$ and $E_{\eta}=0.01$; the dashed curve is for $E_{\eta}=0.001$. The dotted curve is from an OTT calculation with the same $\alpha_{0}$. During the linear phase of the growth, $M(t) \propto \exp (2 \lambda t)$, where $\lambda=2.55$ for OTT; $\lambda=5.50$ for both MTT calculations.

where $\mathcal{V}_{\text {tort }}(s, t)$ is the torsional wave speed (Braginsky 1970):

$$
\mathcal{V}_{\text {tort }}(s, t)=\left(\frac{1}{E_{\eta} \mathcal{A}(s)} \int_{\mathcal{C}(s)} B_{s}^{2}(\boldsymbol{x}, t) \mathrm{d}^{2} x\right)^{1 / 2} \quad \text { or } \quad \mathcal{V}_{\text {tort }}(s, t)=E_{\eta}^{-1 / 2}\left(\frac{\alpha^{*}(s, t)}{\mathcal{A}(s)}\right)^{1 / 2} \text {. }
$$

It is generally true that $\alpha^{*}(s, t) \propto z_{1}^{3}$ as $s \rightarrow 1$ and, since $\mathcal{A}(s) \propto z_{1}, \mathcal{V}_{\text {tort }}(s, t) \propto(1-s)^{1 / 2}$ for $s \rightarrow 1$ by $(4.4 d)$. For dipole parity, $\mathcal{V}_{\text {tort }}(s, t) \propto s$ as $s \rightarrow 0$. A good approximation to the torsional wave speed is $\mathcal{V}_{\text {tort }}(s, t)=\mathcal{V}_{0}(t) s(1-s)^{1 / 2}$, where $\mathcal{V}_{0}(0)=\left(3 / E_{\eta}\right)^{1 / 2} B_{0}$ in the special case $(2.3 a)$. For magnetic fields of quadrupolar parity, $\alpha^{*}(s, t) \propto s$ for $s \rightarrow 0$ so that $\mathcal{V}_{\text {tort }}(0, t)=O(1)$.

As $E_{\eta}$ is so small (of order $4 \times 10^{-10}$, according to $\S 3.1$ ) and $\mathcal{V}_{\text {tort }}(s, t)=O\left(E_{\eta}^{-1 / 2} \mathcal{B}\right.$ ), torsional waves introduce a new short time scale, $\tau_{\text {tort }}=\mathcal{V}_{\text {tort }}^{-1}$, into core dynamics. In dimensional units for Earth's core, $\mathcal{V}_{\text {tort }}(s, t) \approx 2 \times 10^{-2} \mathrm{~m} \mathrm{~s}^{-1}$ and is about $100 \mathcal{U}$; for $\mathcal{B}=25$ gauss, $\tau_{\text {tort }} \approx 5$ yr so that $\tau_{\eta} / \tau_{\text {tort }} \approx 10^{5}$, assuming the dynamo time scale $\tau_{\eta}=r_{o}^{2} / \eta \approx 5 \times 10^{5} \mathrm{yr}$. So there are many torsional oscillations of period $\tau_{\text {tort }}$ during the transition to the steady state of our $\alpha^{2}$ model, but the duration of that transition is $O\left(\tau_{\eta}\right)$, this being the time scale over which ohmic resistance damps out the waves. All our numerical integrations were carried out on a workstation; this made values of $E_{\eta}$ smaller than $10^{-4}$ inaccessible to us. Figure 2 below therefore shows only one oscillation convincingly because $\tau_{\eta} / \tau_{\text {tort }} \approx E_{\eta}^{-1 / 2} \mathcal{B}$, is not large enough to show many. For the $\alpha \omega$ models of paper 2, the final OTT solutions were periodic, with periods that were unrelated to $\tau_{\text {tort }}$, but the MTT evolution to these solutions is accompanied by torsional waves.

The significance of the short time scale of torsional waves was not lost on Taylor (1963, §5), and it seems to us worth quoting a short extract from his paper where 
he recognizes the limitations of OTT that are now removed in MTT. We make minor changes to his text so that it conforms with our notation:

If this constraint is not satisfied then rapid motions occur (in which the acceleration term $\mathrm{D}_{t} \boldsymbol{U}$ cannot be neglected) until such time as the torques producing this motion satisfy $\int_{\mathcal{C}(s)}(\boldsymbol{J} \times \boldsymbol{B})_{\phi} \mathrm{d}^{2} x=0$.

There is, however, a perplexing paradox. It appears, from the numerical results of $\S 5.3$ for the $\alpha^{2}$ model, that there is only one value of the constant $K$ defined in (4.3c) for which $U_{\phi}^{\mathrm{G}}(s, \infty)$ for MTT coincides for all $s$ with $\left[U_{\phi}^{\mathrm{G}}(s, \infty)\right]_{\text {OTT }}$ for OTT. The single exception is $K=\left[U_{\phi}^{\mathrm{G}}(1, \infty)\right]_{\text {OTт }}$ for which the MTT and OTT solutions are the same at infinite $t$. The point is simple: except for that special value of $K$, one cannot require the MTT flow to satisfy $U_{\phi}^{\mathrm{G}}(1, \infty)=K$ and $U_{\phi}^{\mathrm{G}}(1, \infty)=\left[U_{\phi}^{\mathrm{G}}(1, \infty)\right]_{\mathrm{OTT}}$ simultaneously! Section 5.3 below points the way MTT solutions solve the paradox.

At long last, we return from finding $\boldsymbol{U}^{\mathrm{G}}(\boldsymbol{x}, t)\left(=U_{\phi}^{\mathrm{G}}(s, t) \mathbf{1}_{\phi}\right)$ to determining $\boldsymbol{U}^{\mathrm{A}}(\boldsymbol{x}, t)$. It is helpful to cast the defining (4.2a) of MTT in the same form as $(3.7 a)$ for OTT by replacing $\boldsymbol{F}(\boldsymbol{x}, t)$ in $(3.7 a)$ by

$$
\boldsymbol{H}=\boldsymbol{F}-E_{\eta} \partial_{t} U_{\phi}^{\mathrm{G}}(s, t) \mathbf{1}_{\phi} .
$$

Note particularly that $H_{\phi}^{\mathrm{G}}(s, t)=0$, which is analogous to $F_{\phi}^{\mathrm{G}}(s, t)=0$ for OTT; see $(3.7 d)$. This means that the process of solving $(4.2 a, b)$ is identical to that of solving $(3.7 a, b)$, which was achieved by $(3.9 a-e)$. Both processes determine the flow $\boldsymbol{U}^{\mathrm{A}}(\boldsymbol{x}, t)$ at the same time $t$ as they advance $\boldsymbol{B}(\boldsymbol{x}, t)$ (in the case of OTT) or $U_{\phi}^{\mathrm{G}}(s, t)$ (in the case of MTT). This point is elaborated in the next subsection.

\subsection{Discussion; the relevance of $M T T$}

One may ask, 'How can one defend MTT, when it retains only part of the inertial force?' We attempt to answer that question in this subsection in which we return to dimensioned variables.

When the MS equations are integrated, with a time-independent $\alpha(\boldsymbol{x})$ (such as (5.3) below) from an initial state (such as $(2.3 a, b)$ ), ohmic diffusion will ultimately cause $\boldsymbol{B}$ and $\boldsymbol{U}$ to disappear if $\alpha_{0}$ is not large enough. Otherwise $\boldsymbol{B}$ and $\boldsymbol{U}$ will evolve to a steady state, or to a statistically steady oscillatory state. The evolution to this final state is controlled by the three time scales relevant to our project: $\tau_{\eta}, \tau_{\text {tort }}$ and $\tau_{\Omega}$. The longest, $\tau_{\eta}=O\left(r_{o}^{2} / \eta\right) \sim 5 \times 10^{5}$ years, is the principal time scale of dynamo action; $\tau_{\text {tort }}=O\left(r_{o} / V_{\text {tort }}\right) \sim 5$ years is characteristic of the time taken by torsional waves to cross the core; and $\tau_{\Omega}=O\left(\Omega^{-1}\right) \sim 1$ day is a typical time scale of inertial waves when $\boldsymbol{B}=\mathbf{0}$. See Greenspan (1968) and Zhang \& Liao (2017). Neglecting $\boldsymbol{B}$ in describing inertial waves is an oversimplification that tends to underestimate the torsional wave time scale $\tau_{\Omega}$; see e.g. Lan, Kuang \& Roberts (1993). It is made here to simplify the presentation. Two other time scales relevant to core dynamics are (i) the spin-up time, $O\left(E^{-1 / 2} \Omega^{-1}\right) \sim 10^{5} \mathrm{yr}$, but this does not arise in MS because $E=0$. And (ii) the overturning time $r_{o} / \mathcal{U}$, which according to $\S 4$ is approximately 100 times greater than $\tau_{\text {tort }}$ but is still tiny compared with $\tau_{\eta}$. (In MTT, the overturning time is $O\left(\tau_{\text {tort }}\right.$ ).)

In OTT, evolution is dictated by $\partial_{t} \boldsymbol{B}$ which is the only time derivative in the governing equations (the dimensioned $(3.1 c, e)$ and $(3.7 a, b))$, and this imposes the time scale $\tau_{\eta}$ of $\boldsymbol{B}$ through the induction (3.1e). The equation governing $\boldsymbol{U}$ is diagnostic. The evolution of both the geostrophic and ageostrophic parts of $\boldsymbol{U}$ is determined by $\boldsymbol{B}$, and at the same moment in time as $\boldsymbol{B}$, through Taylor's constraint (3.7e). 
In MTT, the governing equations (3.1e) and (4.2a) have 2 time derivatives, $\partial_{t} \boldsymbol{B}$ and $\partial_{t} U_{\phi}^{\mathrm{G}}(s, t)$, associated with the $\tau_{\eta}$ and $\tau_{\text {tort }}$ time scales. Only the ageostrophic part of $\boldsymbol{U}$, associated with the even shorter time scale, $\tau_{\Omega}$, evolves synchronously with $U_{\phi}^{\mathrm{G}}$.

The many studies of rotating fluids, reviewed by Zhang \& Liao (2017) and Greenspan (1968) for $\boldsymbol{B} \equiv \mathbf{0}$, indicate what happens when the inertial force is retained in full. Zhang \& Liao (2017, chap. 5) go to considerable lengths, deriving all possible inertial modes for spherical $\mathrm{v}$ for $\boldsymbol{B}=\mathbf{0}$. There are an infinite number of inertial eigenstates, each with its own frequency, which is bounded above by $2 \Omega$. The longer the $z$-wavelength of a mode, the lower its frequency, which becomes $O\left(\tau_{\text {tort }}^{-1}\right)$ only in the geostrophic limit of $z$-independence. To describe waves dependent on $z$, the inertial force $\rho D_{t} \boldsymbol{U}$ has to be retained in full, while the $z$-independent geostrophic inertial modes can be found by retaining only $\rho \partial_{t} U_{\phi}^{\mathrm{G}} \mathbf{1}_{\phi}$. Likewise, the MTT developments of $\S 4$ succeed because they retain only the $\rho \partial_{t} U_{\phi}^{\mathrm{G}} \mathbf{1}_{\phi}$ part of the inertial force. If more were included, the entire $t$-derivative of $\boldsymbol{U}$ would have to be retained to follow the evolution of $\boldsymbol{U}$ on the very short $\tau_{\Omega}$ time scale, presenting insuperable computational obstacles.

In summary: The OTT of Taylor (1963) filters out all inertial modes so that only the $\tau_{\eta}$ time scale survives. By keeping the geostrophic part (and only the geostrophic part) of the inertial force, MTT retains the $\tau_{\eta}$ and $\tau_{\text {tort }}$ time scales. To retain the inertial force $\rho D_{t} \boldsymbol{U}$ in full, and not merely its geostrophic part, $\rho \partial_{t} U_{\phi}^{\mathrm{G}} \mathbf{1}_{\phi}$, would be tantamount to attempting to reinstate today's numerical dynamos at the inaccessible Ekman number of zero.

\section{Numerical results}

\subsection{Task for $M T T$}

As already mentioned in $\S 2$, we seek only MTT mean-field dynamos in this paper. Although this rescues the torsional waves abandoned by OTT, the inclusion of these waves by MTT is only incidental to our main objective. As we stressed in the last subsection, MTT is the best way of approximating the inertial force while avoiding the large Ekman numbers that are so devastating to the geophysical relevance of today's computer models; see $\S 2$. We now present numerical results for MTT dynamos; these may be compared with the OTT dynamos of paper 2 .

We start by summarizing $\S \S 3$ and 4 . In one respect MTT solutions are simpler to obtain than OTT solution. There are no constraints and $\boldsymbol{U}$ is obtained by time stepping predictive equations. But one disadvantage of MTT is that one has to take more time steps in integrating the governing equations because the torsional wave time scale $\tau_{\text {tort }}=1 / V_{\text {tort }} \propto E_{\eta}^{1 / 2}$ has to be resolved, and when $E_{\eta}$ is small this is much less than the dynamo time scale $\tau_{\eta}=r_{o}^{2} / \eta$.

MTT requires the numerical solution of 4 predictive equations, namely the 3 scalar equations (3.1e) and the modified Taylor condition (4.2a):

$$
\begin{gathered}
\frac{\partial \boldsymbol{B}}{\partial t}=\nabla \times(\boldsymbol{U} \times \boldsymbol{B}+\alpha \boldsymbol{B})+\nabla^{2} \boldsymbol{B}, \quad \nabla \cdot \boldsymbol{B}=0, \\
\frac{\partial U_{\phi}^{\mathrm{G}}(s, t)}{\partial t}=\frac{1}{E_{\eta} \mathcal{A}(s)} \int_{\mathcal{C}(s)}(\boldsymbol{J} \times \boldsymbol{B})_{\phi} \mathrm{d}^{2} x .
\end{gathered}
$$

Equations $(5.1 a, b)$ and the dynamo conditions $(3.1 h, i)$ decide the evolution of $\boldsymbol{B}$; equation (5.1c) and condition (2.2) determine how $U_{\phi}^{\mathrm{G}}$ evolves. At each time step, $\boldsymbol{U}(\boldsymbol{x}, t)$ must be found from $\boldsymbol{F}(\boldsymbol{x}, t)$ and $U_{\phi}^{\mathrm{G}}(s, t)$ by the process described in $\S 4$. 
We integrated $(5.1 a, c)$, starting from

$$
\boldsymbol{B}(\boldsymbol{x}, 0)=B_{0}\left[3 s z \mathbf{1}_{s}+\left(5-6 s^{2}-3 z^{2}\right) \mathbf{1}_{z}\right], \quad U_{\phi}^{\mathrm{G}}(s, 0)=0,
$$

for which $K\left(=U_{\phi}^{\mathrm{G}}(1,0)\right)$ is zero; see $(4.3 c)$. Other $K$ are considered in $\S 5.3$.

As in paper 2, we made Braginsky's (Braginsky (1964)) choice of

$$
\alpha(s, z)=\frac{729}{16} \alpha_{0} r^{7} z\left(1-r^{2}\right)^{2},
$$

where $\alpha_{0}$ is the maximum of $\alpha$. For $\alpha_{0} \geqslant \alpha_{T} \approx 13.80$, the solutions equilibrate to nontrivial steady states; for $\alpha_{0}<\alpha_{T}, \boldsymbol{B}$ eventually decays to zero. For the $\alpha \omega$ dynamos of paper 2, supercritical solutions equilibrate to steadily oscillating dynamos. In $\S 5.3$, we shall compare MTT solutions with the OTT dynamos of paper 2 for $\alpha^{2}$ mean-field models driven by (5.3), and shall also display results for MTT $\alpha \omega$ dynamos.

\subsection{Numerical methods}

We used a semi-discrete method to solve the MTT model. We first discretized the equations in space and obtained a system of ordinary differential equations in time. These were solved using a Runge-Kutta scheme of Shu (1988).

The spatial discretization was carried out on three overlapping grids, which were described in detail in appendix B of Wu \& Roberts (2014). They involved

(i) a 'square' grid covering the region, $-\Delta s \leqslant s \leqslant 0.65,-0.65 \leqslant z \leqslant 0.65$, where $\Delta s$ is the grid spacing in $s$;

(ii) a polar grid covering $0.5 \leqslant r \leqslant 1$ and $-\Delta \theta \leqslant \theta \leqslant \pi+\Delta \theta$, where $\Delta \theta$ is the grid spacing in $\theta$; and

(iii) a cylindrical grid, as specified by Nakajima \& Roberts $(1995 a, b)$, covering the entire computational domain.

There are 4 primary variables in the MTT calculations: $U_{\phi}^{\mathrm{G}}$ and three components of $\boldsymbol{B}$. Other quantities involving $\boldsymbol{U}$ are auxiliary variables that can be obtained from the 4 primary variables; the cylindrical grid was used mainly for that task. The polar grid and the square grid were used to solve the induction equation while enforcing the dynamo conditions $(3.1 h, i)$. (If the polar grid alone had been used, a grid singularity would have occurred at $r=0$. By supplementing the polar grid by the square grid, this difficulty is avoided.)

To preserve the integrity (2.2) of our reference frame, we used the discrete expression of (4.3a) which, for our cylindrical grid, is

$$
E_{\eta} s_{i+1 / 2} \mathcal{A}\left(s_{i+1 / 2}\right) \frac{\partial U_{\phi}^{\mathrm{G}}\left(s_{i+1 / 2}, t\right)}{\partial t}=\frac{\Gamma_{z}\left(s_{i+1}, t\right)-\Gamma_{z}\left(s_{i}, t\right)}{\Delta s},
$$

where $s_{i}$ and $s_{i+1 / 2}$ refer to the integer and half-integer grid values, respectively. Thus the change in the total angular momentum in time becomes

$$
\frac{\mathrm{d}}{\mathrm{d} t}\left(\sum_{i} s_{i+1 / 2} \mathcal{A}\left(s_{i+1 / 2}\right) U_{\phi}^{\mathrm{G}}\left(s_{i+1 / 2}, t\right) \Delta s\right)=\frac{\Gamma_{z}(1, t)-\Gamma_{z}(0, t)}{E_{\eta}}=0 .
$$

The results presented below used the same grid system and the same number of grid points as Wu \& Roberts (2014). The calculations are second-order accurate in 
both space and time. Numerical stability limits the magnitude of the attainable $E_{\eta}$ which, for the grid just specified, is $E_{\eta} \geqslant 10^{-4}$ for the $\alpha^{2}$ model and $E_{\eta} \geqslant 10^{-5}$ for the $\alpha \omega$ model. To reach smaller values of $E_{\eta}$, the number of grid points would have to be increased. We did not analyse the system to determine how the time step is constrained. The time step was found by trial and error guided by the Courant condition and by the constraint imposed by magnetic field diffusion.

\subsection{MTT results for dipolar models}

Most of the figures shown below are for $t$ large enough for the solutions to be close to their final steady or statistically steady states; we term these 'saturated states'. We consider first dipolar $\alpha^{2}$ models and compare them with the OTT dynamos of (2: §5).

Because this paper uses MTT instead of the OTT of paper 2, the evolution of $\boldsymbol{B}$ from $(2.1 b, c)$ differs from that of paper 2: evolution in MTT depends on $E_{\eta}$ but that in OTT does not. This is apparent in figure 2 which shows the internal magnetic energy, $M(t)$, for $\alpha_{0}=14.5$ for 2 values of $E_{\eta}$. Also shown by the dotted curve is the corresponding $M(t)$ from paper 2 for OTT. The 3 curves approach one another as $t$ increases, and each approaches the same steady-state value $M(\infty) \approx 0.128$. The existence of the torsional wave in MTT is evident; $M(t)$ for both MTT solutions overshoots $M(\infty)$ before recovering. In comparison, the $M(t)$ of the OTT solution increases monotonically to $M(\infty)$ (apart from an initial decrease whose origin was explained in paper 2). The OTT solution shown was extracted from paper 2.

Paper 1 pinpointed the way ohmic resistance damps out torsional waves; if there is no source to maintain a torsional wave, resistivity will remove it on the $\tau_{\eta}$ time scale $\left(r_{o}^{2} / \eta\right)$. Our initial non-zero $U_{\phi}^{\mathrm{G}}$ launches a torsional wave but does not maintain it, so its 'half-life' is only approximately $\tau_{\eta}$. Over this period, there will be approximately $\tau_{\eta} / \tau_{\text {tort }}$ maxima and minima of $M(t)$, where $\tau_{\text {tort }} \propto r_{o} / V_{\text {tort }}$, the period of the torsional wave, is proportional to $E_{\eta}^{1 / 2}$ (see $\S 4$ ). In the geophysical case, $\tau_{\eta} / \tau_{\text {tort }} \sim 10^{5}$ according to $\S 4.1$, and the waves traverse the core so many times before being attenuated that the mechanism of their excitation becomes the primary target of theory. Such small $E_{\eta}$ and large $\tau_{\eta} / \tau_{\text {tort }}$ are not practical for dynamo simulations, and therefore far fewer extrema of $M$ can be anticipated. Numerical difficulties increase as $E_{\eta}$ diminishes, and our limited computer resources made it impossible for us to obtain reliable results for $E_{\eta}$ smaller than $10^{-4}$. Figure 2 shows only one maximum of $M$ convincingly. Future MTT models at smaller $E_{\eta}$ should see more.

While $\boldsymbol{B}$ is small, it grows exponentially: $\boldsymbol{B} \propto \mathrm{e}^{\lambda t}$ and the $M(t) \propto \mathrm{e}^{2 \lambda t}$ as shown in figure 2. This may be seen in the 'straight line' segments of the three $M(t)$ of figure 2. During this 'linear phase', $\lambda=2.55$ for the OTT calculation and $\lambda=5.50$ for both MTT cases. The different slopes of the straight line segments of MTT and OTT are apparent in figure 2. They arises because OTT solutions must satisfy Taylor's constraint as $\boldsymbol{B}$ and $M(t)$ grow, but MTT solutions do not. Therefore the $\lambda$ of the MTT solutions coincides with the $\lambda$ of the kinematic dynamo for the assigned $\alpha_{0}=14.5$, but the $\lambda$ of the OTT solution is smaller and vanishes for the marginal $\alpha_{T} \approx 13.8$.

The remaining three figures for the $\alpha^{2}$ dynamos show only the geostrophic part, $U_{\phi}^{\mathrm{G}}(s, t)$, of the MTT flows.

Figure 3 is for $\alpha_{0}=14.5, E_{\eta}=0.01$ and $K\left(=U_{\phi}^{\mathrm{G}}(1,0)\right)=0$; see $(4.3 c)$. It displays $U_{\phi}^{\mathrm{G}}(s, t)$ for $t=34.2,41.1,47.9$ and 54.8. There are 2 minima of $U_{\phi}^{\mathrm{G}}$. The one nearer to $s=1$ is denoted by $\left(U_{\phi}^{\mathrm{G}}\right)_{\min }$ and its location by $s_{\min }$. For $t=34.2,41.1,47.9$ and $54.8,\left(s_{\min },\left(U_{\phi}^{\mathrm{G}}\right)_{\min }\right)=(0.830,-1.635),(0.835,-1.777),(0.842,-1.898)$ and $(0.845,-2.000)$, respectively. In the interval $s_{\min } \leqslant s \leqslant 1, U_{\phi}^{\mathrm{G}}$ increases dramatically 


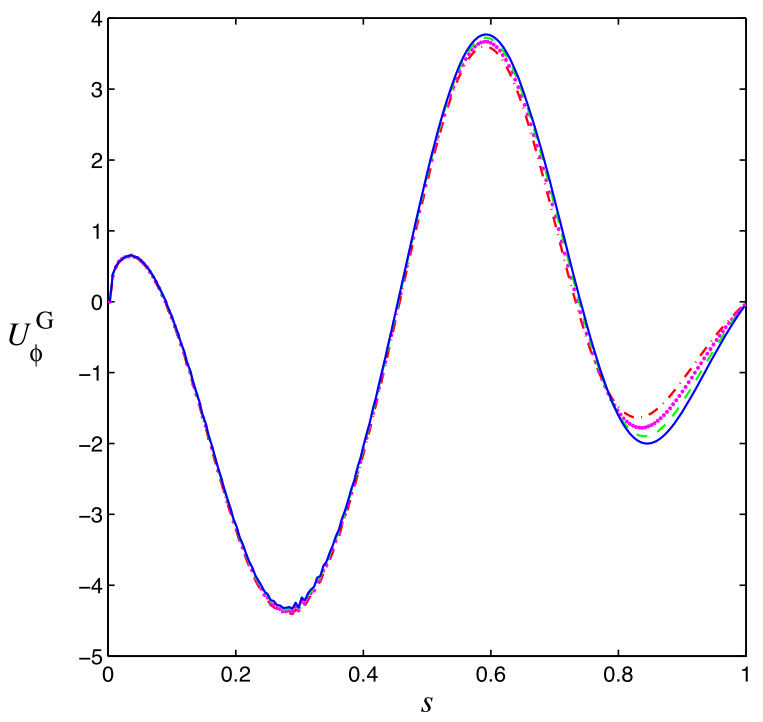

FIGURE 3. The geostrophic flow, $U_{\phi}^{\mathrm{G}}(s, t)$, as a function of $s$ for the MTT $\alpha^{2}$ model at different times; $\alpha_{0}=14.5, E_{\eta}=0.01$ and $U_{\phi}^{\mathrm{G}}(1, t)=0$. The red dashed-dotted curve is for $t=34.2$, the magenta dotted curve is for $t=41.1$, the green dashed curve is for $t=47.9$, and the blue solid curve is for $t=54.8$.

to 0 . As $t$ increases, so does the depth $-\left(U_{\phi}^{\mathrm{G}}\right)_{\min }$ of the minimum and its location $s_{\min }$. This trend suggests that, as $t \rightarrow \infty, s_{\min }$ will tend to 1 and $-\left(U_{\phi}^{\mathrm{G}}\right)_{\min }$ will tend to $-\left(U_{\phi}^{\mathrm{G}}\right)_{\mathrm{OTT}}(1, \infty) \approx 4.8$.

This figure and the next indicate how the paradox of $\S 4$ may be resolved: Because the rate at which $U_{\phi}^{\mathrm{G}}(1, \infty)$ approaches $K$ is proportional to $\mathcal{V}_{\text {tort }}(s, t)$ and vanishes with it as $s \rightarrow 1$, the differences $\boldsymbol{U}(\boldsymbol{x}, t)-[\boldsymbol{U}(\boldsymbol{x}, t)]_{\text {отт }}$ and $\boldsymbol{B}(\boldsymbol{x}, t)-[\boldsymbol{B}(\boldsymbol{x}, t)]_{\text {отт }}$ remain non-zero, becoming simple discontinuities on the equator, $(s, z)=(1,0)$, at $t=\infty$. There they have no dynamic or inductive effect, and do not affect $m_{z}^{\text {total }}$.

Figure 4 re-enforces this interpretation. This figure displays $U_{\phi}^{\mathrm{G}}(s, t)$ as $E_{\eta}$ is reduced for fixed $t=41.1$; here again $\alpha_{0}=14.5$ and $K=0$. Displayed are plots for $E_{\eta}=10^{-2}, 10^{-3}$ and $10^{-4}$. Also shown is the OTT solution for the same $\alpha_{0}$. Employing the same terminology used when describing figure $3, U_{\phi}^{\mathrm{G}}(s, t)$ attains $\left(U_{\phi}^{\mathrm{G}}\right)_{\min }$, which becomes deeper and moves to larger $s$ as $E_{\eta}$ is reduced, while hugging the OTT solution until it leaves it near $s=s_{\min }$ to climb rapidly to the assigned $U_{\phi}^{\mathrm{G}}(1, t)=K=0$. The similarity of the progression of solutions for fixed $t$ and decreasing $E$ is related to the progression of solutions for fixed $E$ and increasing $t$ (figure 3 ) through the $E^{1 / 2}$ time scale of torsional waves identified in $\$ 4$. (The slight irregularity of $U_{\phi}^{\mathrm{G}}(s, t)$ for $E_{\eta}=10^{-4}$ is due to the same numerical difficulty as mentioned above in connection with figure 2.)

Figure 5 shows how $U_{\phi}^{\mathrm{G}}(s, 41.1)$ for MTT solutions changes when $t$ and $E_{\eta}$ are fixed and only $K$ defined in $(4.3 c)$ is varied; as before $\alpha_{0}=14.5$ and $\left.E_{\eta}=0.001\right)$. Displayed are plots for $K=0,-2.0,-4.5$ and -6.0 . That there are substantial differences near $s=1$ where the boundary condition $(4.3 c)$ is enforced is no surprise. The OTT solution, for which $\left[U_{\phi}^{\mathrm{G}}(1)\right]_{\mathrm{OTT}} \approx-4.8$ is also shown. The MTT plots for $K=-4.5$ and -6.0 straddle the OTT solution. This demonstrates consistency between the OTT and MTT solutions. 


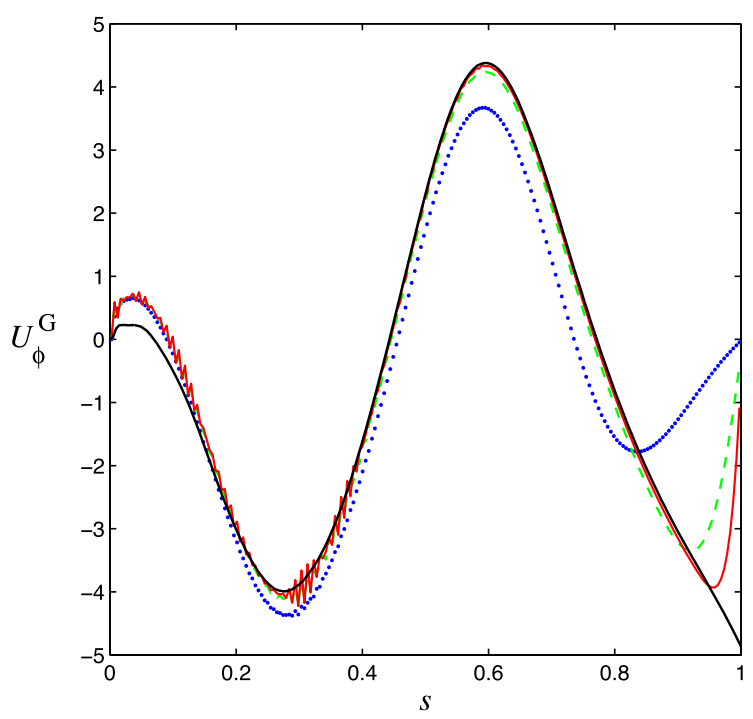

FIgURE 4. The geostrophic flow, $U_{\phi}^{\mathrm{G}}(s, 41.1)$, as a function of $s$ at different $E_{\eta}$ for the MTT $\alpha^{2}$ model; $\alpha_{0}=14.5$ and $U_{\phi}^{\mathrm{G}}(1, t)=0$. The blue dotted curve is for $E_{\eta}=0.01$, the green dashed curve is for $E_{\eta}=0.001$ and the red solid curve is for $E_{\eta}=0.0001$. In addition, the black solid curve is for the OTT model for the same $\alpha_{0}$.

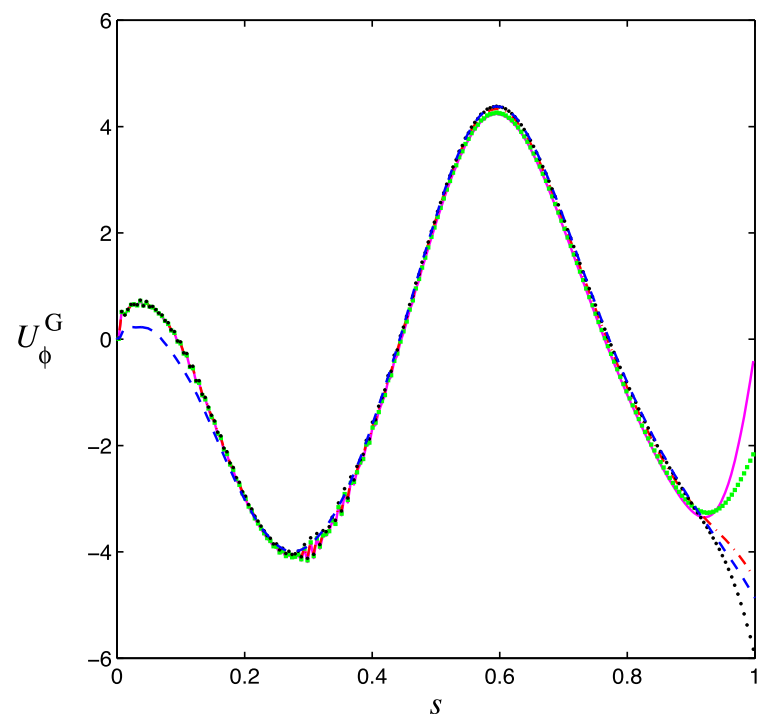

FIgURE 5. The geostrophic flow, $U_{\phi}^{\mathrm{G}}(s, 41.1)$, as a function of $s$ at different $E_{\eta}$ for the MTT $\alpha^{2}$ model; $\alpha_{0}=14.5$ and $E_{\eta}=0.001$. The magenta solid curve is for $U_{\phi}^{\mathrm{G}}(1,0)=0$, the curve of solid green squares is for $U_{\phi}^{\mathrm{G}}(1,0)=-2.0$, the red dashed-dotted curve is for $U_{\phi}^{\mathrm{G}}(1,0)=-4.5$ and the black dotted curve is for $U_{\phi}^{\mathrm{G}}(1,0)=-6.0$. In addition, the blue dashed curve is for the OTT model for the same $\alpha_{0}$; for this OTT model, $U_{\phi}^{\mathrm{G}} \approx-4.8$ at $s=1$. 


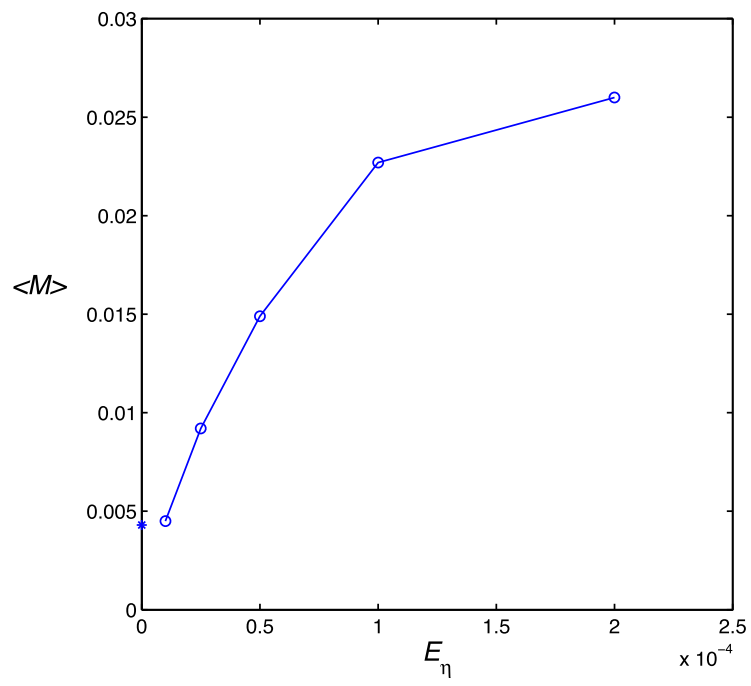

FIGURE 6. The averaged internal magnetic energy, $\langle M\rangle$, plotted in circles as a function of $E_{\eta}$, for saturated MTT $\alpha \omega$ states; $\alpha_{0}\left(=-\omega_{0}^{\prime}\right)=425$. The $\langle M\rangle$ value based on the OTT calculation with the same $\alpha$ and $\omega^{\prime}$ is indicated by the asterisk.
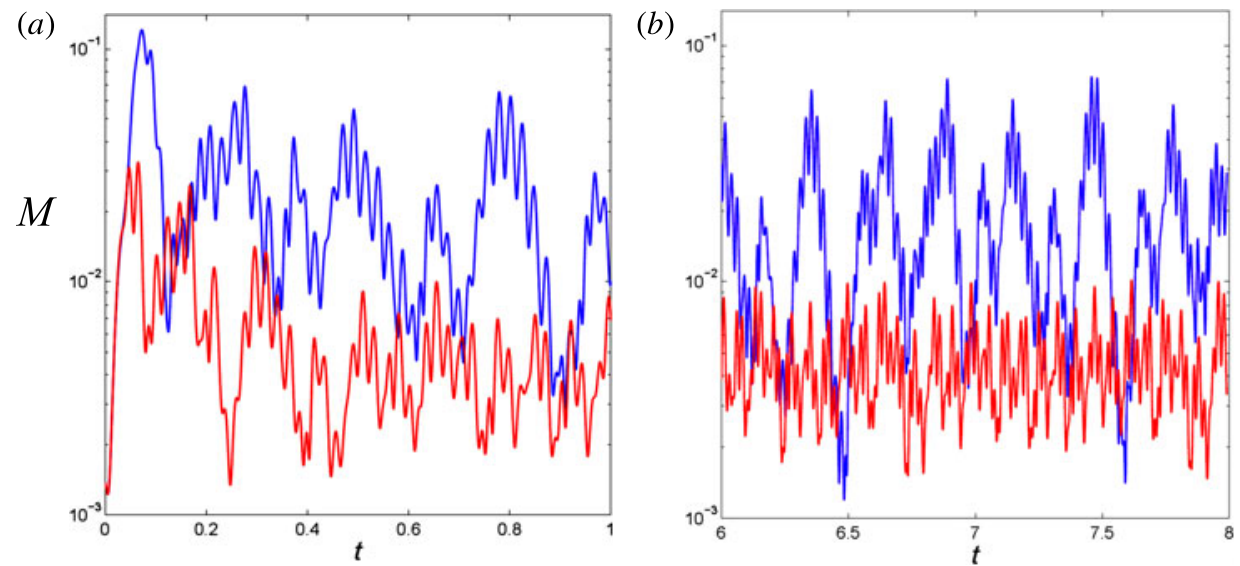

FIgURE 7. Time dependence of the internal magnetic energy, $M(t)$, for the MTT $\alpha \omega$ model with $\alpha_{0}\left(=-\omega^{\prime}\right)=425$ : $(a)$ at an early phase, and $(b)$ in the final saturated state. The blue curves are for $E_{\eta}=10^{-4}$ and the red curves for $E_{\eta}=10^{-5}$.

Our final 3 figures are for MTT $\alpha \omega$ models but we also compare them with OTT $\alpha \omega$ dynamos of (2: $\S 6)$. In all 3 cases, $\alpha_{0}\left(=-\omega_{0}^{\prime}\right)=425$.

Figure 6 shows the mean $\langle M(t)\rangle$ of the evolved internal magnetic energy, $M(t)$, as a function of $E_{\eta}$. The inferred value of $\langle M(t)\rangle \approx 0.004$ for $E_{\eta}=0$ may be compared with the OTT value of $\langle M(t)\rangle \approx 0.0043$ : see $(2: \S 6)$. Interestingly, the amplitude of $\langle M(t)\rangle$ increases with $E_{\eta}$, as the torsional wave speed (4.4d) deceases.

Figure 7 shows $M(t)$ for $E_{\eta}=10^{-4}$ and $E_{\eta}=10^{-5}$ at an early stage in the evolution of the solution from its starting point $(5.2 a, b)$ and in its final evolved state. The increase in the amplitude of $M(t)$ with time is evident. As it is impossible to 


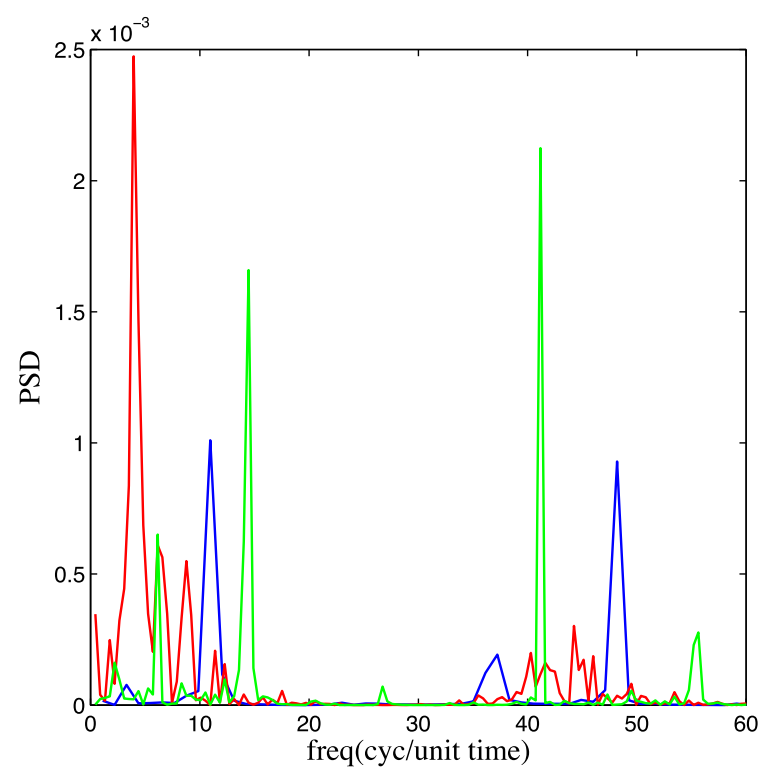

FIGURE 8. The power spectral density (PSD) of the internal magnetic energy, $M(t)$, as a function of frequency for saturated states of the $\alpha \omega$ model with $\alpha_{0}\left(=-\omega^{\prime}\right)=425$ : $(a)$ in red, for MTT and $E_{\eta}=10^{-4} ;(b)$ in green, for MTT and $E_{\eta}=10^{5} ;(c)$ in blue for OTT. In case $(a)$ the PSD has been divided by 40 .

extract quantitative information from this figure, or from (2: figure 14a) for OTT, we performed some spectral analyses:

Figure 8 shows the power spectral density (PSD) of the three $M(t)$ of figure 7 in their saturated states. The spectral peaks for $E_{\eta}=10^{-5}$ are sharper than for OTT and are significantly displaced from them.

\section{Conclusion}

Readers will be well aware of how much this article owes to the solid foundation to magnetostrophy laid by Taylor (1963). Nevertheless, by restoring part of the inertial force, we generalized his concept in paper 1 (Roberts \& Wu 2014). We called the resulting modification of his theory 'modified Taylor theory' (or 'MTT'), and have shown that it admits torsional waves, which are excluded by 'OTT', i.e. the 'original Taylor theory' (Taylor 1963). We have argued in $\S 4.1$ that MTT is the only practical way of improving on OTT. In $\S 5$, we have presented numerical results for MTT $\alpha^{2}$ and $\alpha \omega$ mean-field dynamos. Very recently, Li, Jackson \& Livermore (2018) have presented numerous numerical examples of MTT dynamos. Their paper and this one show that MTT is a practical tool for studying magnetostrophy ('MS'). In turn, and as argued in $\S 2$, we see MS as currently the only numerical route to geophysical realism.

\section{Acknowledgements}

This work was supported by the National Science Foundation under grant EAR1417031. We also are grateful to $\mathrm{Li}$ et al. for giving us the opportunity of studying their paper, prior to its publication. 


\section{REFERENCES}

Abarca Del Rio, R., Gambis, R. \& Salstein, D. A. 2000 Interannual signals in length of day and atmospheric angular momentum. Ann. Geophys. 18, 347-364.

BraginsKy, S. I. 1964 Kinematic models of the Earth's hydromagnetic dynamo. Geomagn. Aeron. 4, 572-583.

BRAGINSKY, S. I. 1965 Self-excitation of a magnetic field during the motion of a highly conducting fluid. Sov. Phys. JETP 20, 726-735; (the original Russian version of this paper appeared before Braginsky (1964)).

BRAGINSKY, S. I. 1970 Torsional magnetohydrodynamic vibrations in the Earth's core and variations in day length. Geomagn. Aeron. 10, 1-8.

Buffett, B. A. 1992 Constraints on magnetic energy and mantle conductivity from the forced nutations of the Earth. J. Geophys. Res. 97, 19581-19597.

Buffett, B. A. \& Christensen, U. R. 2007 Magnetic and viscous coupling at the core-mantle boundary: inferences from observations of the Earth's nutations. Geophys. J. Intl 171, 145-152.

Chandrasekhar, S. 1954 The instability of a layer of fluid heated below and subject to the simultaneous action of a magnetic field and rotation. I. Proc. R. Soc. Lond. A 225, 173-184.

Chandrasekhar, S. 1956 The instability of a layer of fluid heated below and subject to the simultaneous action of a magnetic field and rotation. II. Proc. R. Soc. Lond. A 237, 476-484.

Chandrasekhar, S. 1961 Hydrodynamic and Hydromagnetic Stability. Oxford University Press; Reprinted by Dover publications.

Cowling, T. G. 1933 The magnetic field of sunspots. Mon. Not. R. Astron. Soc. 94, 39-48.

Davies, C. J., Gubbins, D. \& Jimack, P. K. 2011 Scalability of pseudospectral methods for geodynamo simulations. Concurr. Comput. Pract. Exper. 23, 38-56.

Dormy, E. 2016 Strong-field spherical dynamos. J. Fluid Mech. 789, 500-513.

Dormy, E. \& Soward, A. M. (Eds) 2007 Mathematical Aspects of Natural Dynamos, pp. 142-145. Taylor \& Francis.

Eltayeb, I. A. \& Roberts, P. H. 1970 On the hydromagnetics of rotating fluids. Astrophys. J. 162, 699-701.

Gillet, N., Jault, D., Canet, E. \& Fournier, A. 2010 Fast torsional waves and strong magnetic fields within Earth's core. Nature 465 (7294), 74.

Greenspan, H. P. 1968 The Theory of Rotating Fluids. Cambridge University Press.

Hollerbach, R. 1994 Imposing a magnetic field across a nonaxisymmetric shear layer in a rotating spherical shell. Phys. Fluids 6, 2540-2544.

Holme, R. \& De Viron, O. 2013 Characterization and implications of intradecadal variations in length of day. Nature 499 (7457), 202.

Krause, F. 1966 Explanation of stellar and planetary magnetic fields by dynamo action of turbulent motion. In Proc. $4^{\text {th }}$ Consult. Solar Physics and Hydromagn., Sopot, 20-23 Sept., 1966, vol. 77, pp. 157-170. Acta Univ. Wratislava.

Krause, F. \& RÄDleR, K.-H. 1980 Mean-Field Magnetohydrodynamics and Dynamo Theory. Academy Press.

LAN, S., KuAng, W. \& Roberts, P. H. 1993 Ideal instabilities in rapidly rotating MHD systems that have critical layers. Geophys. Astrophys. Fluid Dyn. 69, 133-160.

Li, K., JACKSON, A. \& Livermore, P. W. 2018 Taylor state dynamos found by optimal control: axisymmetric examples. J. Fluid Mech. (to appear).

Livermore, P. W. \& Hollerbach, R. W. 2012 Successive elimination of shear layers by a hierarchy of constraints in inviscid spherical-shell flows. J. Math. Phys. 53, 073104,1-18.

Livermore, P. W., Ierley, G. \& JACKson, A. 2009 The construction of exact Taylor states. I. The full sphere. Geophys. J. Intl 179, 923-928.

Livermore, P. W., Jackson, A. \& Hollerbach, R. W. 2013 Electromagnetically-driven westward drift and inner-core superrotation in Earth's core. Proc. Natl Acad. Sci. USA 110; doi:10.1073/pnas.0709640104.

Moffatt, H. K. 1969 The degree of knottedness of tangled vortex lines. J. Fluid Mech. 35, 117-129.

Moffatt, H. K. 1978 Magnetic Field Generation in Electrically Conducting Fluids. University Press. 
Nakajima, T. \& Roberts, P. H. 1995 a A mapping method for solving dynamo equations. Proc. R. Soc. Lond. A 448, 1-28.

NAKAJIMA, T. \& RoberTs, P. H. $1995 b$ An application of a mapping method to the asymmetric dynamo equations. Phys. Earth Planet. Inter. 91, 53-61.

PARKER, E. N. 1955 Hydromagnetic dynamo models. Astrophys. J. 122, 293-314.

Parker, E. N. 1957 The solar hydromagnetic dynamo. Proc. Natl Acad. Sci. USA 43, 8-14.

PARKer, E. N. 1979 Cosmical Magnetic Fields; Their Origin and Their Activity. Clarendon Press.

Pozzo, M., Davies, C., Gubbins, D. \& Alfé, D. 2012 Thermal and electrical conductivity of iron at Earth's core conditions. Nature 485, 355-358.

Proudman, I. 1956 The almost rigid rotation of viscous fluid between concentric spheres. J. Fluid Mech. 1, 505-516.

RÄDLER, K.-H. 1966 Zur eletrodynamix turbulent bewegter lietender medium. PhD thesis, University of Jena.

Roberts, P. H. \& Aurnou, J. M. 2012 On the theory of core-mantle coupling. Geophys. Astrophys. Fluid Dyn. 106, 157-230.

Roberts, P. H. \& King, E. M. 2013 On the genesis of the Earth's magnetism. Rep. Prog. Phys. 76, 096801.

Roberts, P. H. \& Stix, M. 1971 The turbulent dynamo. A translation of a series of papers by F. Krause, K.-H. Rädler and M. Steenbeck. NCAR Tech. Note TN/IA-60. Available from https://opensky.ucar.edu/islandora/object/technotes\%3A44/datastream/PDF/view, (the translation uses 'helicity' in place of the author's 'Schraubensinn').

Roberts, P. H. \& Wu, C.-C. 2014 On the modified Taylor constraint. Geophys. Astrophys. Fluid Dyn. 108, 696-715; This is referred to above as 'Paper 1'.

Schaeffer, N., Jault, D., NAtaf, H. C. \& Fournier, A. 2017 Turbulent geodynamo simulations: a leap towards Earth's core. Geophys. J. Intl 211, 1-29.

SHU, C. W. 1988 Total-variation-diminishing time discretizations. SIAM J. Sci. Stat. Comput. 9, 1073-1084.

Soward, A. M. 1972 A kinematic theory of large magnetic Reynolds number dynamos. Phil. Trans. R. Soc. Lond. A 272, 431-462.

Soward, A. M. 1974 A convection-driven dynamo I. Weak-field case. Phil. Trans. R. Soc. Lond. A 275, 611-651.

SowARD, A. M. \& Jones, C. A. $1983 \alpha^{2}$-dynamos and Taylor's constraint. Geophys. Astrophys. Fluid Dyn. 27, 87-122.

SowArd, A. M. \& Roberts, P. H. 2014 Eulerian and Lagrangian means in rotating, magnetohydrodynamic flows II. Braginsky's nearly axisymmetric dynamo. Geophys. Astrophys. Fluid Dyn. 108, 269-322.

Steenbeck, M. \& Krause, F. 1966 Erklärung stellarer und planetarer Magnetfelde durch einen turbulenzbedingten Dynamomechanismus. Z. Naturforsch. 21a, 1285-1296; translated as 'Paper III' in Roberts and Stix (1971).

Steenbeck, M., Krause, F. \& RÄDler, K.-H. 1966 Berechnung der mittleren Lorentz-Feldstärke $\overline{\boldsymbol{v} \times \boldsymbol{B}}$ für ein elektrisch leitendes Medium in turbulenter, durch Coriolis-Kräfte beeinflußter Bewegung. Z. Naturforsch. 21a, 369-376; translated as 'Paper II' in Roberts and Stix (1971).

Stewartson, K. 1966 On almost rigid rotations. Part 2. J. Fluid Mech. 26, 131-144.

SweEt, P. A. 1950 The effect of turbulence on a magnetic field. Mon. Not. R. Astron. Soc. 110, 69-83.

TAYLOR, J. B. 1963 The magnetohydrodynamics of a rotating fluid and the Earth's dynamo problem. Proc. R. Soc. Lond. A 274, 274-283.

Teed, R. J., Jones, C. A. \& Tobias, S. M. 2013 The dynamics and excitation of torsional waves in geodynamo simulations. Geophys. J. Intl 196, 724-735.

Teed, R. J., Jones, C. A. \& Tobias, S. M. 2015 The transition to Earth-like torsional oscillations in magnetoconvection simulations. Earth Planet. Sci. Lett. 419, 22-31.

Voĉadlo, L., Alfè, D., Price, G. D. \& Gillan, M. J. 2000 First principles calculations on the diffusivity of Fe-S at experimentally accessible conditions. Phys. Earth Planet. Inter. 120, $145-152$. 
Wicht, J. \& Christensen, U. R. 2010 Torsional oscillations in dynamo simulations. Geophys. J. Intl 181, 1367-1380.

de Wijs, G. A., Kresse, G., Voĉado, L., Dobson, D., Alfè, D., Gillan, M. J. \& Price, G. D. 1998 The viscosity of liquid iron at the physical conditions of the Earth's core. Nature 392, 805-807.

Wu, C.-C. \& Roberts, P. H. 2014 On magnetostrophic mean-field solutions of the geodynamo equations. Geophys. Astrophys. Fluid Dyn. 109, 84-110; This is referred to above as 'Paper 2'.

Zhang, K. \& LiaO, X. 2017 Theory and Modeling of Rotating Fluids. Convection, Inertial waves and Precession. Cambridge University Press. 